\title{
Multilevel mutation-selection systems and set-valued duals
}

July 27,2018

\section{Donald A. Dawson ${ }^{(1)}$}

\begin{abstract}
A class of measure-valued processes which model multilevel multitype populations undergoing mutation, selection, genetic drift and spatial migration is considered. We investigate the qualitative behaviour of models with multilevel selection and the interaction between the different levels of selection. The basic tools in our analysis include the martingale problem formulation for measure-valued processes and a generalization of the function-valued and set-valued dual representations introduced in Dawson-Greven (2014). The dual is a powerful tool for the analysis of the ergodic behaviour of these processes and the study of evolutionary systems which model phenomena including altruism, the emergence of cooperation and more complex interactions.
\end{abstract}

Keywords: Multilevel measure-valued process, multilevel selection, set-valued dual.

\section{AMS Subject Classification: 60J70, 92D25}

\section{This research is supported by NSERC}

1) School of Mathematics and Statistics, Carleton University, Ottawa K1S 5B6, Canada

e-mail: ddawson@math.carleton.ca 


\section{Contents}

Contents 2

1 Introduction

1.1 Hierarchical population structure . . . . . . . . . . . . . .

1.2 Historical remarks on hierarchy in population genetics and evolutionary biology .

1.2.1 A multideme model with two types of individuals . . . . . . . . . . .

1.2.2 A diffusion process model of multilevel selection with two types . . . . . . 5

1.3 Multilevel multitype measure-valued processes . . . . . . . . . . . . . 6

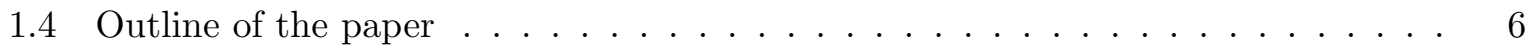

2 Multitype-multilevel mutation-selection models 7

2.1 A two level finite population model . . . . . . . . . . . . . . . . G

2.2 The martingale problem formulation . . . . . . . . . . . . . . 9

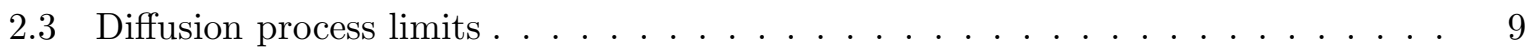

2.3.1 The limiting single deme diffusion process . . . . . . . . . . . . . 10

2.4 Exchangeable system of Wright-Fisher diffusions . . . . . . . . . . . . . . . 10

2.4.1 Deme level fitness functions . . . . . . . . . . . . . . . 10

2.4.2 The limiting generator as $N_{1} \rightarrow \infty$ and $N_{2}<\infty \ldots \ldots \ldots \ldots$

2.5 Empirical measure-valued processes and the Fleming-Viot limit . . . . . . . . 13

3 Duality for interacting and two-level Fleming-Viot systems 15

3.1 A function-valued dual . . . . . . . . . . . . . . . 16

3.2 A set-valued dual for exchangeably interacting systems of Fleming-Viot processes

3.2 .1 The dual representation . . . . . . . . . . . . . . . 19

3.3 A set-valued dual for the two level Fleming-Viot process . . . . . . . . . . 20

3.3.1 The duality relation for the two level Fleming-Viot process and its appli-

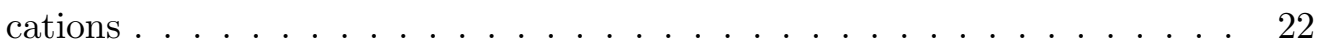

3.3 .2 Moment calculations . . . . . . . . . . . . . . 26

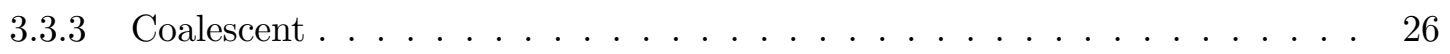

4 Multilevel population systems with two types 27

4.1 Nonlinear measure-valued dynamics with $\gamma_{\mathbf{1}}=\gamma_{\mathbf{2}}=\mathbf{0} \ldots \ldots \ldots \ldots$

4.2 Phase transitions: the role of randomness . . . . . . . . . . . .

4.2 .1 Level I randomness . . . . . . . . . . . . . . . . . . . . . 30

4.2 .2 The case $\gamma_{1}>0, c=0 \ldots \ldots \ldots \ldots \ldots \ldots \ldots$

4.2 .3 Level II randomness $\gamma_{\mathbf{2}}>\mathbf{0} \ldots \ldots \ldots \ldots \ldots \ldots$

5 Multitype multilevel population systems 35

5.1 Equilibria and fixation probabilities . . . . . . . . . . . . . 35

5.2 Examples of multilevel effects . . . . . . . . . . . . . . . .

5.2 .1 A model of cooperation. . . . . . . . . . . . . . . 36

5.2 .2 Emergence of mutualistic types . . . . . . . . . . . . 38 
5.2 .3 A three level system . . . . . . . . . . . . . . . . . 39

5.3 The study of more complex multilevel interactions - set-valued Monte Carlo ap-

proximation ............................. 40

5.4 Extensions . . . . . . . . . . . . . . . . . . . . . . . . . 40

References 40 


\section{Introduction}

Multitype populations are naturally modeled as measure-valued processes. In this paper we consider a class of multilevel measure-valued processes which model ensembles of subpopulations with mutation and selection at the subpopulation level and possible death and replacement of subpopulations. In particular this includes mathematical models of multilevel selection which has been the subject of considerable debate in the evolutionary biology literature. Before introducing our multilevel models we begin with a brief review of some of this literature.

\subsection{Hierarchical population structure}

The hierarchical structure of populations plays a fundamental role in the biological and social sciences. In evolutionary biology and ecology the hierarchy includes ecosystems, community, species, organism, genes and in the social sciences we have cites, regions, nations, etc. These are systems in which at each level of the hierarchy we have a collection of elements of the next lower level in the hierarchy. The description of a unit at a given level in the hierarchy involves the distribution of the different characteristics of the individuals at the next lower level in the hierarchy.

\subsection{Historical remarks on hierarchy in population genetics and evolutionary biology}

Biological evolution can be viewed in terms of a hierarchy of levels of organisation going from the molecular level to the species level and social of groups of members of a species. A natural question is to what extent does the Darwinian mechanism of variation and selection of fitter types in a competitive environment play a role at the various levels.

An early application of group selection was by Wynne-Edwards (1962) [97] who used it to explain adaptations and social behaviour of animals. Subsequently G.C. Williams (1966) [91] made a highly critical analysis of group selection which was very influential. John Maynard Smith (1964),(1976) (53, [54]) considered both group selection and kin selection which was introduced by W.D. Hamilton (1964) [35] and concluded that there may be conditions under which group selection is effective. In the subsequent decades there has been intense debate among evolutionary biologists about the extent to which evolution has been shaped by selective pressures acting at the level of groups of individuals.

In recent years the role of multilevel selection has re-emerged in a number of contexts including the emergence of life (Szathmáry and Demeter [77), structural complexity (Görnerup and Crutchfield (2008) [32]), prebiotic evolution (Hogeweg and Takeuchi [38]), plasmid replication in bacteria (Paulsson (2002) [66]), evolution of cooperation (Traulsen and Nowak [83]) and sociobiology (Wilson and Wilson) [94]. In the study of cultural evolution Boyd and Richardson

[9] suggest that interdemic group selection can be important when there are multiple stable equilibria at the deme level and the emergence of higher level equilibria occurs. Moreover these ideas are relevant in the context of spatially structured populations and evolutionary ecology (see Lion and van Baalen [47, Lion et al [48]). A detailed study of host-pathogen systems 
in the framework of multilevel selection was carried out by Luo (2013) [51], [50] and Luo and Mattingly [52] who demonstrated that a phase transition between the dominance of selection at the different levels can occur as the model parameters are varied. Multilevel selection also underlies current research in the development of complex human societies (see e.g. Turchin et al. 85]). Several books have been written on the question of the levels of selection. These include Brandon and Burian [11, Sober and Wilson [76], Keller [39] and Okasha [64]. An number of other recent research papers on multilevel selection are included in the References.

We end with a quotation of Leigh [44] that provides a useful perspective on these questions: "These conditions ( e.g. he quotes Kimura's conditions - see (4.93)) seem so wonderfully improbable that, following Williams (1966), most biologists have focused almost exclusively on individual selection. Improbability, however, does not mean impossibility. Group selection capable of overwhelming selection within groups, played a crucial role in some major transitions ...".

An objective of this research is to develop tools to identify conditions under which higher level selection is relevant for the class of mathematical models we consider.

\subsubsection{A multideme model with two types of individuals}

In order to introduce the main ideas we briefly review a formulation of group selection given by Aoki [2]). This begins with a countable collection of demes where each deme is a population of $n$ individuals which are either type A or type B. Type B individuals are altruistic and add to the fitness of the deme. The life cycle of a deme involves four discrete events, namely, migration, reproduction, extinction and recolonization. In the reproduction stage, within each deme the population undergoes weighted finite population resampling in which type $\mathrm{B}$ has fitness $-s$ (with $s>0$ ). The probability that a deme suffers extinction is a monotone decreasing function of the proportion of type $\mathrm{B}$ individuals it contains, that is, the fitness of the deme increases as a function of the number of altruistic individuals it contains. In the migration stage a random number of individuals within a deme are replaced by individuals chosen at random from the total population pool. Aoki then obtained a recursion formula for the probability distribution of the number of individuals of type B per deme over successive life cycles and discussed the question

of the long time behavior of this distribution, in particular whether or not the proportion of type B goes to zero or not.

\subsubsection{A diffusion process model of multilevel selection with two types}

The class of Wright-Fisher diffusion processes plays an important role in population genetics. Following Aoki [2]), an analogous extension of the Wright-Fisher process was introduced by Kimura (1983) 40] with alleles $A$ and $B$ distributed in an infinite number of competing demes. It is assumed that $B$ is the altruistic allele which has a selective disadvantage $s_{1}$ but which is beneficial for a deme in competition with other demes, namely, a deme having frequency $x$ for $B$ has advantage $s_{2}\left(x-\int y \nu(d y)\right)$ where $\nu(d y)$ is the distribution of the frequency of type $B$ individuals over the set of demes. This leads to the integro-differential equation for the dynamics of the density $\{\widetilde{\nu}(t, x)\}_{t \geq 0}$ where $\nu(t, d x)=\widetilde{\nu}(t, x) d x$ : 


$$
\frac{\partial \widetilde{\nu}(t, x)}{\partial t}=\frac{\gamma_{1}}{2} \frac{\partial^{2}}{\partial x^{2}}(x(1-x) \widetilde{\nu}(t, x))-\frac{\partial}{\partial x}(M(t, x) \widetilde{\nu}(t, x))+s_{2}\left(x-\int y \widetilde{\nu}(t, y) d y\right) \widetilde{\nu}(t, x)
$$

where

$$
M(t, x)=m_{21}(1-x)-m_{12} x+c\left(\int y \widetilde{\nu}(t, y) d y-x\right)-s_{1} x(1-x),
$$

and $m_{12}, m_{21}$ are the mutation rates $1 \rightarrow 2,2 \rightarrow 1$ respectively, $c$ is the rate of migration between colonies and the resampling rate $\gamma_{1}$ is inversely proportional to the effective population size at a deme. This model will be discussed in detail in subsection 4.2.1 including Kimura's analysis which was based methods of ordinary differential equations as well as the analysis using the dual representation which will be developed in section 3 . The duality method is not restricted to twotype systems (as is the case for the ode method) and can be used to study general multitype systems.

\subsection{Multilevel multitype measure-valued processes}

The natural framework for multilevel multitype models with random effects at different levels is the setting of multilevel measure-valued processes. Models of this type were first developed for multilevel branching systems in Dawson and Hochberg [19] and Etheridge [26]. The long-time behaviour of multilevel measure-valued processes is investigated in $\mathrm{Wu}$ [96], Dawson and Wu [25], and Gorostiza, Hochberg and Waklbinger [33]. In particular two-level measure-valued processes have state spaces of the form $\mathcal{M}(\mathcal{M}(E))$ for some Polish space $E$ where $\mathcal{M}(E)$ denotes the space of Borel measures on $E$. In this paper we work with an analogous class of two level probability measure-valued processes formulated in terms of a well-posed martingale problem which generalizes the Kimura model to systems with more than two types, more complex interactions and to the diffusion limit of systems with finitely many demes.

\subsection{Outline of the paper}

The main objectives of this paper are to formulate a general measure-valued framework for multilevel population systems with mutation and selection and to develop the method of duality for multilevel measure-valued stochastic processes with applications to population systems with multilevel selection. In section 2 we introduce the class of models characterized as solutions to a well-posed martingale problem. In Section 3 we introduce the dual processes used to establish that the martingale problems are well-posed and to compute joint moments. These are given by the multilevel generalization of the class of function-valued and set-valued dual processes introduced in Dawson and Greven [24]. In Section 4 we consider the long-time behavior of systems with two types and with two levels of selection. In Section 5 we introduce some more complex models of systems with $K \geq 2$ types and with multilevel selection as well as further possible extensions of these models and methods. 


\section{Multitype-multilevel mutation-selection models}

\subsection{A two level finite population model}

We begin with a two-level finite population model given by an exchangeably interacting system of Moran particle systems with selection at both levels. We assume that the higher level fitness of a subpopulation can depend on the distribution of level I types within the subpopulation. In other words at the group level the fitness $V_{2}(\mu)$ of a subpopulation described by its distribution $\mu$ over the space of types, is a result of a network of interactions. Then the resulting distribution of the collection of subpopulations $\left\{\mu_{i}\right\}_{i \in S}$ is formulated in the setting of multilevel measurevalued processes which provide a natural setting for the study of hierarchical systems of this type.

We begin with a simple colony containing $N_{1}$ individuals described by a Moran model. Each individual has type in $\mathbb{I}=\{1, \ldots, K\}$. We let

$$
\begin{aligned}
& n_{k}:=\text { number of individuals of type } k \in\{1, \ldots, K\} \\
& N_{1}=\sum_{k=1}^{K} n_{k}
\end{aligned}
$$

and we think of the normalized vector $\frac{1}{N_{1}}\left(n_{1}, \ldots, n_{K}\right)$ as an element of $\mathcal{P}(\mathbb{I})$, the space of probability measures on $\{1, \ldots, K\}$,

$$
X=\frac{1}{N_{1}} \sum_{k=1}^{K} n_{k} \delta_{k},
$$

where the single atom measure $\delta_{k}$ represents an individual of type $k$.

The dynamics of a simple colony is given by a continuous time Markov chain, $\left\{X_{t}: t \geq 0\right\}$ with state space $\mathcal{P}(\mathbb{I})$.

The dynamics includes:

- Mutation: given by transition rates $\left\{m_{i j}\right\}_{i, j \in \mathbb{I}}$, that is, the rate at which an individual of type $i$ is replaced by an individual of type $j$

- Sampling: at rate $\frac{\gamma_{1}}{2}$ an individual of type $i$ is replaced by an individual of type $j$ where $j$ is chosen from the empirical distribution $X$

- Selection with fitness function $V_{1}: \mathbb{I} \rightarrow[0,1]$ and intensity $s_{1}$.

The resulting transitions for the probability-measure-valued process are given by

$$
\begin{aligned}
& \mu \rightarrow \mu-\frac{1}{N_{1}} \delta_{i}+\frac{1}{N_{1}} \delta_{j} \text { at rate } m_{i j} \mu(i) \\
& \mu \rightarrow \mu-\frac{1}{N_{1}} \delta_{i}+\frac{1}{N_{1}} \delta_{j} \text { at rate }\left(\left(N_{1}-1\right) \frac{\gamma_{1}}{2}+s_{1} V_{1}(j)\right) \mu(i) \mu(j)
\end{aligned}
$$


for $i, j \in \mathbb{I}$. Note that these assumptions result in a rate of change $d X(t, i) / d t$ due to mutation and selection of order $1 / N_{1}$ which turns out to be the same as the order of the sampling fluctuations when $\gamma_{1}>0$. This corresponds to the case of weak selection in population genetics and the diffusion limit below will involve a time speed-up by a factor of $N_{1}$. In population genetics the parameter $\gamma_{1}$ is viewed as inverse effective population size (see Remark 5.4 in [17]) and is a measure of the population size in relation to the selection intensity in the finite population model.

We now consider a collection of $N_{2}$ colonies (demes) each consisting of $N_{1}$ individuals with internal dynamics within each colony given as above. In addition (as in the Aoki model) there is an interaction between colonies via migration. To model this, individuals within a colony die and are replaced at rate $c>0$ by a new individual with type given by that of a randomly chosen individual in a randomly chosen colony.

The final mechanism is death (extinction) and replacement of colonies following the same mechanism as the sampling mechanism within colonies. That is, a colony dies and is replaced by a copy of a randomly chosen colony. In addition we can include deme-level selection using a level II fitness function $V_{2}(\mu)$ and selection intensity $s_{2}$. For example, we can take a linear fitness function of the form

$$
V_{2}(\mu)=\int v_{2}(x) \mu(d x)
$$

We then consider the empirical measure

$$
\Xi_{N_{2}, N_{1}}(t):=\frac{1}{N_{2}} \sum_{i=1}^{N_{2}} \delta_{\mu_{i}(t)} \in \mathcal{P}(\mathcal{P}(\mathbb{I}))
$$

where $\mu_{i}$ denotes the state of the ith colony, namely,

$$
\mu_{i}=\frac{1}{K} \sum_{j=1}^{K} \frac{n_{i, j}}{N_{1}} \delta_{j}
$$

and $n_{i, j}$ denotes the number of type $j$ individuals in the ith colony.

The resulting transitions due to the level one dynamics are of the form

$$
\nu \rightarrow \nu+\frac{1}{N_{2}}\left(\delta_{\mu-\frac{\delta_{i}}{N_{1}}+\frac{\delta_{j}}{N_{1}}}-\delta_{\mu}\right)
$$

at rate $m_{i j} \mu(i)+\left(\left(N_{1}-1\right) \frac{\gamma_{1}}{2}+s_{1} V_{1}(j)\right) \mu(i) \mu(j) \nu(d \mu)$

The resulting transitions due to level II sampling and selection for the measure-valued process are given by:

$$
\nu \rightarrow\left(\nu+\frac{1}{N_{2}}\left(-\delta_{\mu_{1}}+\delta_{\mu_{2}}\right)\right) \text { rate }\left(s_{2} V_{2}\left(\mu_{2}\right)+\frac{\gamma_{2}}{2}\left(N_{2}-1\right)\right) \nu\left(d \mu_{1}\right) \nu\left(d \mu_{2}\right)
$$

We can then consider the limiting behaviour as $N_{1}$ or $N_{2}$ go to $\infty$, or we can allow them to go to infinity simultaneously with for example $N_{2}=\eta N_{1}$. In the following subsections we will first consider the limit as $N_{1} \rightarrow \infty$ for a finite system of $N_{2}$ demes leading to a system of interacting 
Fisher-Wright diffusions. We then consider the exchangeable system of Fisher-Wright diffusions with additional death and random replacement of demes as described above and then obtain a two level measure-valued diffusion process (called the two level Fleming-Viot model) by letting $N_{2} \rightarrow \infty$.

\subsection{The martingale problem formulation}

The framework in which we specify the different stochastic models is the class of probabilityvalued Markov processes $X(t) \in \mathcal{P}\left(E_{1}\right)$ where $E_{1}$ is a Polish space. Let $D_{E_{1}}([0, \infty)),\left(C_{E_{1}}([0, \infty))\right)$ denote the class of càdlàg (resp. continuous) functions from $[0, \infty)$ to $E_{1}$. We denote by $\left\{\mathcal{F}_{t}\right\}_{t \geq 0}$ the natural filtration of $\sigma$-algebras on these spaces. The probability law $P \in \mathcal{P}\left(D_{E_{1}}([0, \infty))\right)$ is said to be a solution of the martingale problem with generator $(G, D(G))$, where $G$ is a linear operator on $D(G) \subset C\left(E_{1}\right)$ and $D(G)$ is measure-determining on $E_{1}$, if

$$
M_{F}(t):=\quad F(X(t))-\int_{0}^{t} G F(X(s)) d s
$$

is an $\mathcal{F}_{t}$-adapted $P$ martingale for all $F \in D(G)$.

The martingale problem method is used to characterize stochastic processes of interest in many applications. The method (which we will also use below) consists of four steps:

(1) to construct a sequence of approximating processes with probability laws $P_{n} \in \mathcal{P}\left(D_{E_{1}}([0, \infty))\right)$ that satisfy some simple martingale problems,

(2) to show that the laws of the processes are tight, that is, relatively compact in $\mathcal{P}\left(D_{E_{1}}([0, \infty))\right)$

(3) to show limit points of the $P_{n}$ satisfy the martingale problem defined by $(G, D(G)$ ), and

(4) to prove that there is a unique solution to this martingale problem thus characterizing the limiting probability law $P$ of the process of interest. We will use this method to define the Fleming-Viot process that models selection at two levels.

A key tool used to establish the uniqueness of solutions is duality. This is achieved by constructing a dual process $\mathcal{G}_{t}$ with state space $E_{2}$ and function $F: \mathcal{P}\left(E_{1}\right) \times E_{2} \rightarrow \mathbb{R}$ such that the functions $\left\{F(\cdot, g), g \in E_{2}\right\}$ are in $D(G)$ and are measure-determining on $E_{1}$, and the duality relation:

$$
E_{X(0)}\left(F\left(X(t), \mathcal{G}_{0}\right)\right)=E_{\mathcal{G}_{0}}\left(F\left(X(0), \mathcal{G}_{t}\right)\right)
$$

is satisfied for all $\mathcal{G}_{0} \in E_{2}$ for all $X(0) \in \mathcal{P}\left(E_{1}\right)$ (where the right side denotes the expectation with respect to the law of the $\mathcal{G}_{t}$ process). The class of set-valued dual processes we use in the study of multilevel mutation selection systems is developed in detail in Section 3. In addition to using the dual to establish that the martingale problem has a unique solution it will be used below to compute moments, to obtain fixation probabilities and to prove ergodicity.

For general background on the martingale problem formulation see [15], [17] and [27].

\subsection{Diffusion process limits}

In this subsection we will identify the limit of the process $\left\{\Xi_{N_{2}, N_{1}}(t)\right\}_{t \geq 0}$ as $N_{1} \rightarrow \infty$ for fixed $N_{2}<\infty$. 


\subsubsection{The limiting single deme diffusion process}

We first consider the special case in which $N_{2}=1$ and let $N_{1} \rightarrow \infty$.

Proposition 2.1 The limit as $N_{1} \rightarrow \infty$ of the single deme $\left(N_{2}=1\right)$ normalized empirical measure with diffusion scaling leads (with time speed-up $\left.t \rightarrow N_{1} t\right)$ ) to a $K$-type Fleming-Viot process (equivalently, a finite type Wright-Fisher diffusion) which is characterized as the unique solution of the martingale problem given with generator

$$
\begin{aligned}
G^{0} f(\mathbf{x}) & =\sum_{i=1}^{K}\left(\sum_{j=1}^{K}\left(m_{j i} x_{j}-m_{i j} x_{i}\right)\right) \frac{\partial f(\mathbf{x})}{\partial x_{i}} \quad \text { mutation } \\
& +s_{1} \sum_{i=1}^{K} x_{i}\left(V_{1}(i)-\sum_{k=1}^{K} V_{1}(k) x_{k}\right) \frac{\partial f(\mathbf{x})}{\partial x_{i}} \quad \text { selection } \\
& +\frac{\gamma_{1}}{2} \sum_{i, j=1}^{K} x_{i}\left(\delta_{i j} x_{j}-x_{j}\right) \frac{\partial^{2} f(x)}{\partial x_{i} \partial x_{j}} \quad \text { genetic drift }
\end{aligned}
$$

defined on the class $D\left(G^{0}\right)$ given by the class of functions $f$ with continuous second derivatives on the simplex $\Delta_{K-1}=\left\{\left(x_{1}, \ldots, x_{K}\right), x_{i} \geq 0, \sum x_{i}=1\right\}$.

Proof See for example [15], Theorem 2.7.1 for the neutral case and 15] Theorem 10.2.1 for the proof of uniqueness for the case with selection. (Also see [27], Chapter 10, Theorem 1.1 for the derivation of the diffusion limit starting with a discrete generation model.)

\subsection{Exchangeable system of Wright-Fisher diffusions}

We now consider a system of demes labeled by $S=\left\{1,2, \ldots, N_{2}\right\}$ where the population at each deme undergoes mutation and selection as in the single deme process but in addition individuals can migrate between demes at rate $c$ and the population in a deme can become extinct at rate $s_{2}$ and be replaced with population $\mu$ sampled from the empirical distribution of deme compositions. With selection, the replacement deme type is chosen with weights proportional to the level II fitness

$$
0 \leq V_{2}(\mu) \leq 1, \quad \mu \in \mathcal{P}(\mathbb{I})
$$

\subsubsection{Deme level fitness functions}

In order to incorporate fitness at the deme level we must introduce an appropriate class of fitness functions. It is natural to assume that the fitness of a deme (subpopulation) is a function of the distribution of level I types within the deme given by $V_{2}(\mu)$ when the distribution of types within the deme is $\mu \in \mathcal{P}(\mathbb{I})$. We also assume that $V_{2}$ is a bounded and continuous function of $\mu$ (in the topology of weak convergence). Without loss of generality (by the addition of a constant if needed) we can assume that $V_{2}(\mu) \geq 0$. 
Example 1 Consider the special case $\mathbb{I}=\{1,2\}$, and

$$
\left.V_{2}(\mu)\right)=f(\mu(1)) \geq 0
$$

Then (see e.g. Lorentz (1963) 49] (Chapt. 1, Theorem 4)), we can uniformly approximate $V_{2}$ using Bernstein polynomials as follows

$$
V_{2}(\mu)=\lim _{n \rightarrow \infty} \sum_{k, \ell} a_{n, k}(\mu(1))^{k}(\mu(2))^{n-k}
$$

where the coefficients $a_{n, k} \geq 0$.

In general, given a compact Polish space $E$ we consider the space $\mathcal{P}(E)$ of probability measures on $E$ with the topology of weak convergence. We then consider the Bernstein operators $B^{K}$ : $C(\mathcal{P}(E)) \rightarrow C(\mathcal{P}(E)$ ) (where $C(\mathcal{P}(E)$ ) is a normed space with the supremum norm) defined by

$$
B^{K} f(\mu)=\int \ldots \int f\left(\frac{1}{K} \sum_{i=1}^{K} \delta_{x_{i}}\right) \mu\left(d x_{1}\right) \ldots \mu\left(d x_{K}\right)
$$

Then by (Dawson and Gärtner 21] Theorem 3.9) for any $f \in C(\mathcal{P}(E))$

$$
B^{K} f \rightarrow f \quad \text { in } C(\mathcal{P}(E)) \text {. }
$$

This means that we can approximate any bounded continuous fitness function $V_{2} \in C_{+}(\mathcal{P}(\mathbb{I}))$ by

$$
B^{K} V_{2}(\mu)=\int \ldots \int h\left(x_{1}, \ldots, x_{K}\right) \mu\left(d x_{1}\right) \ldots \mu\left(d x_{K}\right)
$$

where $h$ is a bounded non-negative function on $(\mathbb{I})^{K}$. This can be rewritten in the form

$$
B^{K} V_{2}(\mu)=\sum_{i} s_{i, K} \int_{(\mathbb{I})^{K}} \prod_{j=1}^{K} 1_{A_{K, i, j}} d \mu^{\otimes K}
$$

where for each $i$ the $A_{K, i, j}$ are subsets of $\mathbb{I}$. We denote the class of fitness functions of the form (2.12) by $\mathcal{V}_{K}$ and note that we can approximate any bounded continuous fitness function by a functions in $\mathcal{V}:=\cup_{K} \mathcal{V}_{K}$.

Example 2 Types $\mathbb{I}=\{1,2\}$. If $\mu(1)=p_{1}, \mu(2)=p_{2}$, then

$$
\begin{gathered}
V_{2}(\mu)=p_{1} p_{2}, \quad\left(1-V_{2}(\mu)\right)=p_{1}^{2}+p_{2}^{2}+p_{1} p_{2} \\
V_{2}(\mu)=\mu^{\otimes}(C), 1-V_{2}(\mu)=\mu^{\otimes}\left(C^{c}\right)
\end{gathered}
$$

where $C=\{1\} \otimes\{2\}$.

Example 3 Consider the 3 type case $\mathbb{I}=\{1,2,3\}$ with fitness functions as follows:

$$
V_{1}(1)=s_{1}, \quad V_{2}(\mu)=s_{2} \mu(2) \mu(3) .
$$


Example 4 Model with 3 types $\mathbb{I}=\{1,2,3\}$ and mutualistic (state-dependent) fitness.

- $V_{1}(1, \mu)=s_{1} \mu(2), V_{1}(2, \mu)=s_{1} \mu(1), V_{1}(3)=1 / 2$

- Level II fitness is $V_{2}(\mu)=s_{2}\left[\frac{1}{2} \mu(3)+2 s_{1} \mu(1) \mu(2)\right]$.

This can be analysed using the set-valued dual as indicated in Remark 5 .

Example $5 V_{2}(\mu)$ is positive iff the population contains a certain set of properties (from a finite set).

$$
\begin{gathered}
V_{2}(\mu)=\sum e_{i} \mu^{\otimes}\left(A_{i}\right) \\
1-V_{2}(\mu)=\sum e_{i} \mu^{\otimes}\left(A_{i}^{c}\right)
\end{gathered}
$$

where $e_{i} \geq 0, \sum e_{i}=1, A_{i} \subset(\mathbb{I})^{\mathbb{N}}$.

\subsubsection{The limiting generator as $N_{1} \rightarrow \infty$ and $N_{2}<\infty$}

The generator for the resulting model of $N_{2}$ interacting demes: for $F \in C^{2}\left(\mathcal{P}(\mathbb{I})^{N 2}\right)$, with $\mathbf{X}:=\left(\mathbf{x}_{1}, \ldots, \mathbf{x}_{N_{2}}\right) \in(\mathcal{P}(\mathbb{I}))^{N 2}$

$$
\begin{aligned}
& G^{N_{2}, \text { int }} F(\mathbf{X}) \\
& =\eta \sum_{\xi=1}^{N_{2}} G_{\xi}^{0} F(\mathbf{X}) \quad \text { mutation-selection dynamics at each site } \\
& +c \cdot \sum_{\xi=1}^{N_{2}}\left[\sum_{j=1}^{K}\left(\sum_{\xi^{\prime}=1}^{N_{2}} \frac{1}{N_{2}} x_{j}\left(\xi^{\prime}\right)-x_{j}(\xi)\right) \frac{\partial F(\mathbf{X})}{\partial x_{j}(\xi)}\right] \text { migration } \\
& \left.+s_{2} \sum_{\xi=1}^{N_{2}}\left(\frac{1}{N_{2}} \sum_{\xi^{\prime}=1}^{N_{2}} V_{2}(\mathbf{x}(\xi)) F\left(\Phi_{\xi \xi^{\prime}} \mathbf{X}\right)-F(\mathbf{X})\right]\right) \text { deme replacement } \\
& +\frac{1}{2} \gamma_{2} \sum_{\xi=1}^{N_{2}} \sum_{\xi^{\prime}=1}^{N_{2}}\left[F\left(\Phi_{\xi \xi^{\prime}} \mathbf{X}\right)-F(\mathbf{X})\right] \quad \text { deme resampling }
\end{aligned}
$$

where $\Phi_{\xi \xi^{\prime}} \mathbf{X}=\left(\mathbf{x}_{1}, \ldots, \mathbf{x}_{\xi}, \ldots, \mathbf{x}_{\xi}, \mathbf{x}_{N_{2}}\right)$ (corresponding to the replacement of $\mathbf{x}_{\xi}^{\prime}$ by $\mathbf{x}_{\xi}$ ) and $\eta$ is a parameter that depends on the relation between the natural time scales at the two levels.

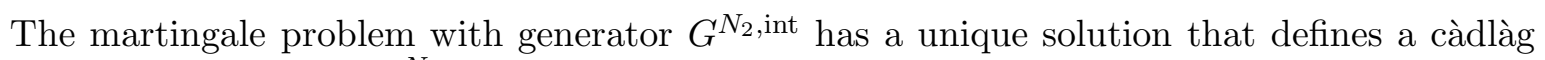
strong Markov process $\left\{\mathbf{X}_{t}^{N_{2}}\right\}_{t \geq 0}$ with state space $(\mathcal{P}(\mathbb{I}))^{N_{2}}$. The proof follows as in the proof of Proposition 2.1 but where the dual process needed to show that the martingale problem is well posed in given in Subsection 3.1. 


\subsection{Empirical measure-valued processes and the Fleming-Viot limit}

We will next consider the limit as $N_{2} \rightarrow \infty$ in the general case in which we can have $s_{2}>0$ and/or $\gamma_{2}>0$. We assume that the initial state satisfies $\left(\mu_{1}(0), \ldots, \mu_{N_{2}}(0)\right)$ is exchangeable.

Lemma 2.2 Consider the Markov process $\mathbf{X}(t)=\left(\mathbf{x}_{1}(t), \ldots, \mathbf{x}_{N_{2}}(t)\right) \in(\mathcal{P}(\mathbb{I}))^{N_{2}}$ with generator $G^{N_{2} \text {,int }}$. Assume that the probability distribution of $\mathbf{X}(0)$ is exchangeable (i.e the distribution is invariant under permutations of $\left.\left\{1,2, \ldots, N_{2}\right\}\right)$. Then $\left(\mathbf{x}_{1}(t), \ldots, \mathbf{x}_{N_{2}}(t)\right)$ is an exchangeable system of $\mathcal{P}(\mathbb{I})$-valued diffusions.

Proof This follows since the migration and level II selection terms in the generator are invariant under permutation - see 86] for the general case of exchangeable diffusions.

The level II empirical process is defined by

$$
\Xi_{t}^{N_{2}}:=\frac{1}{N_{2}} \sum_{j=1}^{N_{2}} \delta_{\mu_{j}} \in \mathcal{P}(\mathcal{P}(\mathbb{I}))
$$

Then by Lemma 2.2, $\Xi^{N_{2}}(t)$ is a $\mathcal{P}(\mathcal{P}(\mathbb{I})$ )-valued Markov process with generator inherited from the interacting system. To describe this we consider the algebra of functions, $D\left(G^{N_{2}}\right)$, on $\mathcal{P}(\mathcal{P}(\mathbb{I}))$ containing functions of the form

$$
H(\nu)=\prod_{k=1}^{K}\left[\int h_{k}\left(\mu_{k}\right) \nu\left(d \mu_{k}\right)\right]
$$

where

$$
h_{k}(\mu)=\sum_{j} h_{k, j} \mu^{\otimes}\left(\prod_{i} 1_{A_{k, i j}}\right) \quad \text { that is, a polynomial on } \mathcal{P}(\mathbb{I}) .
$$

We then define the generator in terms of the generator of the interacting system as follows:

$$
G^{N_{2}} H(\nu):=G^{N_{2}, \text { int }} F\left(\left(\mu_{1}, \ldots, \mu_{N_{2}}\right)\right)
$$

where $H \in D\left(G^{N_{2}}\right)$ and

$$
\nu=\frac{1}{N_{2}} \sum_{j=1}^{N_{2}} \delta_{\mu_{j}},
$$

and $G^{N_{2} \text {,int }}$ is given by $(2.17)$. 
Theorem 1 (29], 24]).

Assume that $\gamma_{2} \geq 0$ and $V_{2} \in \mathcal{V}$. Then

$$
\left\{\Xi_{t}^{N_{2}}\right\}_{t \in[0, T]} \Rightarrow\left(\Xi_{t}\right)_{t \in[0, T]} \text { as } N_{2} \rightarrow \infty
$$

where $\Xi_{t}(d x) \in C_{\mathcal{P}(\mathcal{P}(\mathbb{I}))}([0, T])$ is the two level Fleming-Viot process with level two selection given by the unique solution, $\left\{P_{\nu}: \nu \in \mathcal{P}(\mathcal{P}(\mathbb{I}))\right\}$, to the well-posed $\left(G_{2}, D_{2}\right)$ martingale problem where the domain $D_{2} \subset C(\mathcal{P}(\mathcal{P}(\mathbb{I})))$ consists of the algebra of functions containing functions of the form (2.19) and the generator acting on $D_{2}$ is given by

$$
\begin{aligned}
G_{2} H(\nu) & =\int_{\mathcal{P}(\mathbb{I})} \eta G^{0} \frac{\delta H(\nu)}{\delta \nu(\mu)} \nu(d \mu) \\
& +c \int_{\mathcal{P}(\mathbb{I})} \int_{\mathbb{I}}\left(\frac{\delta}{\delta \mu_{1}(x)} \frac{\delta H(\nu)}{\delta \nu\left(\mu_{1}\right)}\left[\int \nu\left(d \mu_{2}\right) \mu_{2}(d x)-\mu_{1}(d x)\right]\right) \nu\left(d \mu_{1}\right) \\
& +\frac{\gamma_{2}}{2} \int_{\mathcal{P}(\mathbb{I})} \int_{\mathcal{P}(\mathbb{I})} \frac{\delta^{2} H(\nu)}{\delta\left(\nu\left(\mu_{1}\right)\right) \delta\left(\nu\left(\mu_{2}\right)\right)}\left(\nu\left(d \mu_{1}\right) \delta_{\mu_{1}}\left(d \mu_{2}\right)-\nu\left(d \mu_{1}\right) \nu\left(d \mu_{2}\right)\right) \\
& +s_{2}\left[\int_{\mathcal{P}(\mathbb{I})} \frac{\delta H(\nu)}{\delta \nu\left(\mu_{1}\right)}\left[V_{2}\left(\mu_{1}\right)-\int_{\mathcal{P}(\mathbb{I})} V_{2}\left(\mu_{2}\right) \nu\left(d \mu_{2}\right)\right] \nu\left(d \mu_{1}\right)\right],
\end{aligned}
$$

where $G^{0}$ is given by (2.8).

Proof We follow the standard argument which involves three steps: proof of the tightness of the laws of the processes, proof of convergence of the generators on a sufficiently large class of functions and finally proof that the martingale problem associated with the limiting generator has a unique solution. The first two steps follow in the usual way (e.g. proof of [15], Theorem 5.3.1). It then remains to prove the uniqueness - this will be proved in the next section after introducing the appropriate class of dual processes.

Remark 1 An alternative class of functions, $\mathcal{D}_{2}$, is the linear span of functions of the form

$$
H(\nu)=\prod_{k=1}^{K}\left(\int_{\mathcal{P}(\mathbb{I})}\left(\int_{\mathbb{I}^{n_{k}}} h\left(x_{k, 1}, \ldots, x_{k, n_{k}}\right) \mu_{k}^{\otimes n_{k}}\left(d x_{k}\right)\right) \nu\left(d \mu_{k}\right)\right) .
$$

We also consider the convex set $\widetilde{\mathcal{D}}_{2}$ of $[0,1]$-valued functions which contain functions of the above form with $h$ having values in $[0,1]$. Note that this class uniquely determines probability measures on $\mathcal{P}(\mathcal{P}(\mathbb{I}))$. 
Remark 2 The multilevel Fleming-Viot process is the analogue of the multilevel superprocess - see e.g. Dawson-Hochberg (1991) [19], Etheridge (1993) [20], Wu (1994) [96], GorostizaHochberg-Wakolbinger (1995) [33], Dawson-Hochberg-Vinogradov (1996) [20, Dawson and Wu (1996) 203], Dawson-Gorostiza-Wakolbinger (2004) 203.

Remark 3 In the special case $s_{2}=0$ and $\gamma_{2}=0$ we obtain the mean-field limit. We consider a tagged colony - by exchangeability this can be colony $1, \mu_{1}^{N_{2}}(t)$. Then as $N_{2} \rightarrow \infty$ in the limit we obtain the measure-valued McKean-Vlasov dynamics (cf. 24]) given by the solution to the martingale problem with nonlinear generator

$$
G_{1}^{\nu} F(\mu)=G^{0} F(\mu)+c \int_{\mathbb{I}} \frac{\delta F(\mu)}{\delta \mu(x)}\left[\int_{\mathcal{P}(\mathbb{I})} \nu(d \mu)(\mu(d x))-\mu(d x)\right]
$$

and the law of the process, $\Xi_{t}=\mathcal{L}\left(\mu_{t}\right) \in \mathcal{P}(\mathcal{P}(\mathbb{I}))$ is the weak solution of a nonlinear second order partial differential equation.

If we assume $\gamma_{2}=0$ but $s_{2}>0$, then $\Xi_{t}$ is still deterministic and is the solution of a nonlinear second order partial differential equation which is a generalization of Kimura's equation (1.1). Depending on the functions $V_{1}, V_{2}$ and with recombination these nonlinear equations can exhibit a range of behaviors including multiple equilibria and possible periodic or chaotic behaviour (see Akin [1]). In the general case with $\gamma_{2}>0$ we obtain a two level Fleming-Viot process.

\section{Duality for interacting and two-level Fleming-Viot systems}

In this section we introduce a basic tool, namely the generalization of the class of set-valued dual introduced in 24] to the class of two level probability-measure-valued processes $\Xi(t) \in \mathcal{P}(\mathcal{P}(\mathbb{I}))$ which were obtained in the previous section. These processes satisfy the martingale problem with generator $G_{2}$.

$$
\begin{aligned}
M_{H}(t):=\quad & H\left(\Xi_{t}\right)-\int_{0}^{t} G_{2} H\left(\Xi_{s}\right) d s \\
& \text { is a } P-\text { martingale for all } H \in D_{2}\left(G_{2}\right) .
\end{aligned}
$$

The dual process developed here will be used to prove that there is a unique law $P \in \mathcal{P}\left(C_{\mathcal{P}(\mathcal{P}(\mathbb{I}))}([0, \infty))\right)$ which satisfies the martingale problem $(3.25)$. As explained above the idea is to find a dual process $\mathcal{G}_{t}^{2}$ and to establish the duality relation

$$
\left.E_{\Xi(0)}\left(F\left(\Xi(t), \mathcal{G}_{0}^{2}\right)\right)=E_{\mathcal{G}_{0}^{2}}\left(F(\Xi(0)), \mathcal{G}_{t}^{2}\right)\right) .
$$

We begin by obtaining the dual for the system of interacting $\mathcal{P}(\mathbb{I})$-valued processes with generator $G^{N_{2}, \text { int }}$ given by (2.17). For detailed background on the duality method to be used refer to [24] Chapter 5.

In 


\subsection{A function-valued dual}

We now introduce a function-valued dual for the process with generator $G^{N_{2}, i n t}$. The state space for the function-valued dual is the set of functions, $\mathbb{H}$, of the form $\sum_{k} \prod_{i=1}^{N_{2}} \prod_{j=1}^{n_{i}} h_{k, i, j}\left(x_{i j}\right)$. By inspection of the action of the generator $G^{N_{2}, \text { int }}$ on functions in $\mathbb{H}$, we can read off the corresponding function-valued transitions corresponding to mutation, selection and migration as follows.

- Level I Selection with $V_{1}(x)=1_{B}(x)$.

Transitions at rate $s_{1}$

$$
h\left(x_{1}, \ldots, x_{n}\right) \rightarrow 1_{B}\left(x_{i}\right) h\left(x_{1}, \ldots, x_{n}\right)+1_{B^{c}}(x n+1) h\left(x_{1}, \ldots, x_{n}\right)
$$

- Mutation

$$
h\left(\ldots, x_{i}, \ldots\right) \rightarrow \int h(\ldots, y, \ldots) M\left(x_{i}, d y\right)
$$

- Level I Coalescence: At rate $\frac{\gamma n(n-1)}{2}$,

$$
h\left(x_{1}, \ldots, x_{n}\right) \rightarrow h\left(x_{i}, \ldots, x_{i}, \ldots, x_{j-1}, x_{i}, x_{j+1}, \ldots, x_{n}\right)
$$

- Migration: For each $i, j \in S$, at rate $\frac{c}{N_{2}}$,

$$
h_{1}\left(x_{i 1}, x_{i 2}\right) h_{2}\left(x_{j 1}, x_{j 2}\right) \rightarrow h_{1}\left(x_{i 1}, x_{j 3}\right) h_{2}\left(x_{j 1}, x_{j 2}\right)
$$

Here the first index indicates the deme and the second the rank at the given deme.

- Level II selection: By (2.12) and taking convex combinations it suffices to consider a level II fitness function of the form:

$$
\begin{gathered}
V_{2}(\mu)=\mu(B) \\
h(\cdot) \longrightarrow V_{2}(\cdot) h(\cdot)+\left(1-V_{2}(\cdot)\right) \otimes h(\cdot)
\end{gathered}
$$

Then the level II selection: for each $i, j \in S$ in transitions at rate $\frac{s_{2}}{N_{2}}$

$$
h\left(x_{i 1}, x_{i 2}\right) \rightarrow 1_{B}\left(x_{i 1}\right) h\left(x_{i 2}, x_{i 3}\right)+\left(1-1_{B}\left(x_{j 1}\right)\right) h\left(x_{i 1}, x_{i 2}\right)
$$

- Level II coalescence: for each pair $i, j$ at rate $\frac{\gamma_{2}}{2}$

$$
h_{1}\left(x_{i 1}, x_{i 2}\right) h_{2}\left(x_{j 1}, x_{j 2}\right) \rightarrow h_{1}\left(x_{i 1}, x_{i 2}\right) h_{2}\left(x_{i 3}, x_{i 4}\right) .
$$




\subsection{A set-valued dual for exchangeably interacting systems of Fleming-Viot processes}

We now introduce the set-valued dual which will be used to study the interacting system of Fleming-Viot processes and then the limiting two-level Fleming-Viot process. This is based on the set-valued dual introduced in [24] (subsections 9.4, 9.5) for the system of exchangeably interacting Fleming-Viot processes but extended in order to include level II selection and resampling.

We begin with the population at a set of demes labeled by $S$ with

$$
S=\left\{1, \ldots, N_{2}\right\}
$$

with migration between demes as defined in subsubsection 2.3.1 with the assumption of exchangeability. Recall that the state space for the finite system of interacting Fleming-Viot processes is $(\mathcal{P}(\mathbb{I}))^{S}$. The set-valued dual is a refinement of the function-valued dual sketched above. Noting that it suffices to work with linear combinations of indicator function the Level I function-valued and set-valued version of the above dual were introduced and studied in depth in Dawson and Greven [24].

We now introduce the state space and notation needed to define the set-valued dual $\mathcal{G}_{t}$. Recall that $\mathbb{I}=\{1, \ldots, K\}$. We indicate the indicator function of a subset $A \subset \mathbb{I}$ by $1_{A}=$ $\left(e_{1}, \ldots, e_{K}\right)$ with $e_{i}=1$ if $i \in A$ and $e_{i}=0$ is $i \in A^{c}$, that is, the complement of $A$. For example, the indicator function of $\{1,2\} \subset\{1,2,3\}$ is indicated by (110). We sometimes identify finite subsets with their indicator functions.

Let

$$
\begin{aligned}
\mathcal{I}:= & \text { algebra of subsets of } \mathbb{I}^{\mathbb{N}} \\
& \text { of the form } A \otimes_{1} \mathbb{I}^{\mathbb{N}}, A \text { is a subset of } \mathbb{I}^{m}, m \in \mathbb{N},
\end{aligned}
$$

the coordinates in a product in $\mathbb{I}^{m}$ are called ranks. Given $A, B \subset \mathbb{I}$ we denote the product of these sets in $\mathbb{I} \times \mathbb{I}$ as $A \otimes_{1} B$. Given $A, B \subset \mathcal{I}$ we denote the product of these sets in $\mathcal{I} \times \mathcal{I}$ as $A \otimes_{2} B$.

The state space: $\left.\right|^{\mathrm{N}_{2}}$ for the set-valued dual associated to the interacting systems of Fleming-Viot processes with $S=\left\{1, \ldots, N_{2}\right\}$ is the algebra of sets containing sets of the form

$$
\begin{aligned}
& \bigotimes_{2, i \in S}\left(\otimes_{1, j=1}^{n_{i}} A_{i, j}\right), \quad A_{i, j} \in \mathbb{I}, n_{i} \in \mathbb{N}, \\
& \in(\mathcal{I})^{\otimes_{2} S} .
\end{aligned}
$$

In order to describe the dual dynamics we first describe the transitions that occur for a set written as a disjoint union of sets of the form (3.34) where in $A_{i, j} \subset \mathbb{I}$ the first subscript denotes the deme and the second subscript denotes the rank at the deme, and $V_{1}(x)=1_{B}(x)$ with rate $s_{1}>0$. 
The transitions of the set-valued process $\mathcal{G}_{t}^{N_{2}, \text { int }}$ are obtained by restricting the function-valued transitions to indicator functions of sets in $\mathrm{I}^{\mathrm{N}_{2}}$. These are then given by:

Level I selection at rank $j^{*}$ at deme $i^{*} \in S$ at rate $s$ :

$$
A_{i^{*} . j^{*}} \rightarrow B_{i^{*} . j^{*}} \cap A_{i^{*} . j^{*}} \cup B_{i^{*} . j^{*}}^{c} \otimes_{1} A_{i^{*} . j^{*}+1}
$$

and all other ranks larger than $j^{*}$ are also shifted to the right at deme $i^{*}$.

Mutation at rank $j$ at deme $i$ : (refer to [24], Definition 5.12 and Subsubsection 9.5.3)

$$
A_{i j} \subset \mathbb{I} \rightarrow A_{i j} \cup\{\ell\} \text { with } \ell \in \mathbb{I} \text { at rate } \sum_{k \in A} m_{\ell, k},
$$

or

$$
A_{i j} \rightarrow A_{i j} \backslash\{\ell\} \text { at rate } \sum_{k \in A_{i j}^{c}} m_{\ell, k}
$$

Coalescence at rate $\gamma_{1} / 2$ of ranks $j_{1}$ and $j_{2}>j_{1}$ at deme $i \in S: A_{i, j_{1}} \otimes_{1} A_{i, j_{2}} \rightarrow \widetilde{A}_{i, j_{1}}=A_{i, j_{1}} \cap A_{i, j_{2}}$ and $\widetilde{A}_{i, j}=A_{i, j+1}$ for $j \geq j_{2}$.

Migration at rate $\frac{c}{N_{2}}$ of rank $j$ from deme $i_{2} \in S$ to $i_{1} \in S$. Let $A_{i}=\otimes_{1, i=1}^{n_{i}} A_{i j}$.

$$
A_{i_{1}} \otimes_{2} A_{i_{2}} \rightarrow \widetilde{A}_{i_{1}} \otimes_{2} \widetilde{A}_{i_{2}}
$$

with

$$
\begin{gathered}
\widetilde{A}_{i_{1}, n_{1}+1}=A_{i_{2}, j} \\
\widetilde{A}_{i_{2}, \ell}=A_{i_{2}, \ell+1} \quad \text { for } \ell \geq j \\
\widetilde{A}_{i_{2}, \ell}=A_{i_{2}, \ell} \quad \text { for } \ell<j
\end{gathered}
$$

Remark 4 Note that in the limit $N_{2} \rightarrow \infty$ the measure $\nu$ is nonatomic and migration or level II selection transitions always lead to a new (that is unoccupied) deme.

Coupling: Note that every set in $\mathrm{I}^{\mathrm{N}_{2}}$ can be written as the union of a finite number of disjoint sets of the form $\otimes_{2, i=1}^{N} \otimes_{1, j=1}^{n_{i}} A_{i, j}$ with $A_{i, j} \subset \mathbb{I}$ and $N \in \mathbb{N}$. Finally the above transitions are simultaneously carried out in this disjoint union of products and are coupled as follows: all selection, mutation, coalescence and migration operations are simultaneously applied to each rank at each deme of each product in the disjoint union. Each such transition preserves the decomposition of the disjoint union into a new disjoint union - this is obviously satisfied for mutation, coalescence and migration and true for selection in view of the specific form (3.35). 


\subsubsection{The dual representation}

We now state the duality relation between the system of interacting Fleming-Viot processes $\mathbf{X}$ under the assumption

$$
\mathbf{X}(0)=\mu^{\otimes_{2} N_{2}} \text {. with } \mu \in \mathcal{P}(\mathbb{I})
$$

which implies that we have a system of exchangeably interacting Fleming-Viot processes.

Define the function $F: \mathcal{P}(\mathbb{I}) \otimes I \rightarrow[0,1]$ by

$$
F(\mathbf{X}, \mathcal{G})=\mathbf{X}^{*}(\mathcal{G})
$$

where if $\mathbf{X}(0)=\otimes_{2, j=1}^{N_{2}} \mu_{j}$, then $\mathbf{X}^{*}(0)=\otimes_{2, j=1}^{N_{2}}\left(\mu_{j}\right)^{\otimes_{1} \mathbb{N}} \in \mathcal{P}\left(\left(\mathbb{I}^{\mathbb{N}}\right)^{N_{2}}\right)$. For example, if $G=$ $\bigotimes_{i \in S} G_{i}$ with $G_{i} \in \mathcal{I}$, then

$$
\mathbf{X}^{*}(G)=\prod_{j \in S} \mu_{j}^{\otimes_{1} \mathbb{N}}\left(G_{j}\right)
$$

Theorem 2 Let $\mathbf{X}^{N_{2}}$ denote a solution to the martingale problem with generator $G^{N_{2}, \text { int }}$. Then (a) Dual Representation

$$
E_{\mathbf{X}(0)}\left(F\left(\mathbf{X}_{t}^{N_{2}}, \mathcal{G}_{0}^{N_{2}, i n t}\right)\right)=E_{\mathcal{G}_{0}^{N_{2}, i n t}}\left(F\left(\mathbf{X}_{0}^{N_{2}}, \mathcal{G}_{t}^{N_{2}, i n t}\right)\right)
$$

(b) The representation (3.45) uniquely determines the marginal distribution of the process $\mathbf{X}^{N_{2}}(t)$ and therefore establishes the uniqueness of the solution to the martingale problem.

Proof The proof in the case $s_{2}=0$ is given in detail in 24] based on verifying that the generators of the two processes acting on the function $F$ satisfy the relation

$$
G^{N_{2}, i n t} F(\mu, A)=G^{\text {dual }} F(\mu, A) \quad \text { for all } \mu \in \mathcal{P}(\mathbb{I}), A \in \mathbf{I},
$$

where $G^{d u a l}$ is the generator of the set-valued Markov jump process with transition rates given above. The extension to the case $s_{2}>0$ follows in the same way and will be given in more detail for the two-level process below.

\section{Remark 5 State-dependent fitness}

We can also consider level I selection that is state dependent, that is in which the fitness of a type depends on the distribution of types (e.g. diploid). For example we could have the fitness of type 1 proportional to the population proportion of type $B$, that is, $V(1, \mu)=s \mu(B), B \subset \mathbb{I}, s \geq$ 0 . In this case the dual has function-valued transitions at rate $s$ :

$$
\begin{aligned}
f & \rightarrow 1_{B} \otimes_{1}\left[1_{1} f-1_{1} \otimes_{1} f\right]+f \\
= & 1_{B} \otimes_{1}\left[1_{1} f+\left(1-1_{1}\right) \otimes_{1} f\right] \\
& +\left(1-1_{B}\right) \otimes_{1} f .
\end{aligned}
$$


A second example to a set-valued dual which can be used to analyse such systems, eg. mutualistic types (see Example 母). Consider $\mathbb{I}=\{1,2,3\}$.

$$
V_{1}(1)=v_{1}, \quad V_{1}(2, \mu)=v_{M} \cdot \mu(3), V_{1}(3, \mu)=v_{M} \cdot \mu(2),
$$

with transitions

$$
\begin{aligned}
& f \rightarrow 1_{1} f+\left(1_{2}+1_{3}\right) \otimes_{1} f \quad \text { at rate } v_{1}, \\
& f \rightarrow 1_{3} \otimes_{1}\left[1_{2} f+\left(1_{1}+1_{3}\right) \otimes_{1} f\right]+\left(1_{1}+1_{2}\right) \otimes_{1} f \quad \text { at rate } v_{M}, \\
& f \rightarrow 1_{2} \otimes_{1}\left[1_{3} f+\left(1_{1}+1_{2}\right) \otimes_{1} f\right]+\left(1_{1}+1_{3}\right) \otimes_{1} f \quad \text { at rate } v_{M} .
\end{aligned}
$$

\subsection{A set-valued dual for the two level Fleming-Viot process}

The objective of this subsection is to extend the set-valued dual of subsection 3.2 in order to construct set-valued duals $\mathcal{G}_{t}^{2, N_{2}}, \mathcal{G}_{t}^{2}$ for the $\mathcal{P}\left(\mathcal{P}(\mathbb{I})\right.$ )- valued processes $\left\{\Xi^{N_{2}}(t)\right\}_{t \geq 0}$ (assuming exchangeable initial configuration) and $\{\Xi(t)\}_{t \geq 0}$. We assume that the level II selection rate is $s_{2}$ and with fitness function $V_{2}$ and the level II resampling rate is $\frac{\gamma_{2}}{2}$. To simplify the notation we take $\eta=1$ in (2.17) in the subsequent discussion.

The state space: $\mathrm{I}^{2 *}$ for the set-valued dual $\mathcal{G}_{t}^{2}$ is is the algebra of subsets of $\left(\mathbb{I}^{\mathbb{N}}\right)^{\mathbb{N}}$ containing sets of the form

$$
\begin{aligned}
& \bigotimes_{2, i \leq m}\left(\otimes_{1, j=1}^{n_{i}} A_{i, j}\right), \quad A_{i, j} \in \mathbb{I}, n_{i} \in \mathbb{N}, \\
& \in(\mathcal{I})^{\otimes_{2} m}, \quad m \in \mathbb{N},
\end{aligned}
$$

where $i$ is the index of the deme and $j$ is the index of the rank within the deme. The transitions of the dual due to level I mutation, resampling, and selection at each deme and migration between demes are given as above in subsection 3.2. In the $N_{2} \rightarrow \infty$ limit, migrants always move to a new (unoccupied) deme, namely the the unoccupied deme of lowest index.

We assume that $V_{2}$ belongs to the class of level II fitness functions of the form

$$
V_{2}(\mu)=\sum_{j} s_{2, j} V_{2, j}(\mu), \quad V_{2, j}(\mu)=\mu^{\otimes}\left(1_{B_{j}}\right)
$$

and $B_{j}=\prod_{i=1}^{n_{j}} B_{j i}$ with $B_{j i} \subset \mathbb{I}$.

We now introduce the additional transitions that occur due to Level II selection and coalescence. For the former, using the linearity it suffices to describe the contribution to the dynamics of $V_{2, j}$ with $V_{2, j}(\mu)=\mu^{\otimes}(B), B \in \mathcal{I}$.

As above we use the notation $\otimes_{1}$ and $\otimes_{2}$ to distinguish such products on $\mathbb{I}$ and $\mathcal{I}$. Similarly for measures in $\nu_{i} \in \mathcal{P}(\mathcal{P}(\mathbb{I}))$ we write $\nu_{1} \otimes_{2} \nu_{2}$ for the product measure.

$\underline{\text { Set-valued transitions - deme level selection }}$ 
Using the linearity it suffices to consider the contribution to the dynamics of $V_{2, j}$ with $V_{2, j}(\mu)=\mu^{\otimes}\left(B_{j}\right), B_{j} \in(\mathbb{I})^{n_{j}}$. Given such a fitness function and sets $A_{i} \in \mathcal{I}$ with the subscript indicating the deme, then for every $k, \ell \in S$ the action of selection on deme $k$ results in the transition

$$
\bigotimes_{2, i \in S} A_{i} \rightarrow\left(B_{k} \otimes_{1} A_{k} \otimes_{2} \bigotimes_{2, i \neq k} A_{i}\right) \bigcup\left(B_{\ell}^{c} \otimes_{2} \bigotimes_{2, i \in S} A_{i}\right)
$$

occurs with rate $\frac{s_{2, j}}{N_{2}}$ if $S=\left\{1, \ldots, N_{2}\right\}$.

If $S=\mathbb{N}$, then this becomes

$$
\bigotimes_{2, i=1}^{n} A_{i} \rightarrow\left(B_{k} \otimes_{1} A_{k} \otimes_{2} \bigotimes_{2, i \neq k} A_{i}\right) \bigcup\left(B_{\ell}^{c} \otimes_{2} \bigotimes_{2, i=1}^{n} A_{i}\right)
$$

where $A_{i}, B_{i} \in \mathcal{I}, n$ is the number of occupied demes (i.e. not identically $\mathbb{I}^{\mathbb{N}}$ ) and $\ell$ denotes the first unoccupied deme, occurs with rate $s_{2, j}$.

Note that exchangeability is preserved by the dynamics and we can again couple the corresponding indices after a level II selection event, that is all further transitions are applied simultaneously to the corresponding indices (deme and rank at the deme). For this reason we can rewrite (3.51) as

$$
\bigotimes_{2, i=1}^{n} A_{i} \rightarrow\left(B_{k} \otimes_{1} A_{k} \otimes_{2} \bigotimes_{2, i \neq k} A_{i}\right) \bigcup\left(B_{k}^{c} \otimes_{2} \bigotimes_{i} \widetilde{A}_{i}\right)
$$

where $\widetilde{A}_{i}=A_{i}$ if $i<k, \widetilde{A}_{i}=A_{i+1}$ if $i \geq k$. This means that the new event is a disjoint union of events and this is preserved by all further transitions.

Example: Let $\mathbb{I}=\{1,2\}$ with fitness $V_{2}(\mu)=\mu(1)$. Then given $1_{\mathcal{G}}=1_{1}$, then the first transition (in terms of indicator functions)

$$
1_{1} \rightarrow 1_{1} \otimes_{1} 1_{1}+1_{1} \otimes_{2} 1_{2}
$$

so that letting

$$
E_{\nu}(\mu(1))=\int \mu(1) \nu(d \mu)
$$

we have

$$
E_{\nu}(\mu(1)) \rightarrow E_{\nu}(\mu(1))+E_{\nu}\left((\mu(1))^{2}\right)-\left(E_{\nu}(\mu(1))\right)^{2}=E_{\nu}(\mu(1))+\operatorname{Var}_{\nu}(\mu(1))
$$

so that if $\nu$ is not a single atom probability measure the level II selection is effective.

$\underline{\text { Set-valued transitions - deme level coalescence }}$ 
The level II resampling results in the coalescence of two demes, for example demes 1,2 as follows:

$$
\begin{aligned}
& \left(\bigotimes_{1, j} B_{j}\right)_{1} \otimes_{1}\left(\bigotimes_{1, i} A_{i}\right)_{1}+\left(\bigotimes_{1, j} B_{j}\right)_{1}^{c} \otimes_{2}\left(\bigotimes_{1, i} A_{i}\right)_{2} \\
& \rightarrow\left(\bigotimes_{1, j} B_{j}\right)_{1} \otimes_{1}\left(\bigotimes_{1, i} A_{i}\right)_{1}+\left(\bigotimes_{1, j} B_{j}\right)_{1} \otimes_{1}\left(\bigotimes_{1, i} A_{i}\right)_{1} \\
& =\left(\bigotimes_{1, i} A_{i}\right)_{1}
\end{aligned}
$$

where the exterior subscripts denote the deme.

When there is no level II coalescence and $\nu_{0}$ is non-random, the $\{t \rightarrow \nu(t)\}$ is deterministic and it suffices to consider $k_{0}=1$. The reason is that there is no interaction between the supports of the associated set-valued processes starting with disjoint supports in this case so that $\operatorname{Var}\left(\int h(\mu) \nu_{t}(d \mu)\right)=0$.

\subsubsection{The duality relation for the two level Fleming-Viot process and its applica- tions}

We now present the dual representation for the two level Fleming-Viot systems.

Let $\{\Xi(t)\}_{t \geq 0}$ denote a $\mathcal{P}(\mathcal{P}(\mathbb{I}))$-valued process with probability law

$$
P^{\Xi_{0}}=\mathcal{L}(\Xi) \in \mathcal{P}\left(C_{\mathcal{P}(\mathcal{P}(\mathbb{I})))}([0, \infty))\right)
$$

which satisfies the $\left(G_{2}, D_{2}\right)$-martingale problem. Then the time-marginals $\Xi(t)$ are random probability measure on $\mathcal{P}(\mathbb{I})$. Then by de Finetti's theorem (see [15], Theorem 11.2.1) there exist a sequence $\left\{\widetilde{\mu}_{n}\right\}$ of $\mathcal{P}(\mathbb{I})$-valued exchangeable random variables such that $\left(\widetilde{\mu}_{1}, \ldots, \widetilde{\mu}_{n}\right)$ has joint distribution

$$
P^{(n)}\left(t, d \widetilde{\mu}_{1}, \ldots, d \widetilde{\mu}_{n}\right)=\int_{\mathcal{C}_{\mathcal{P}(\mathcal{P}(\mathbb{I}))}[0, \infty)} \Xi\left(t, d \mu_{1}\right) \ldots \Xi\left(t, d \mu_{n}\right) d P^{\Xi_{0}}, \quad n \in \mathbb{N},
$$

that correspond to the moment measures of $\Xi(t)$.

Let $\mathcal{G}_{t}$ with values in ${ }^{2 *}$ denote the set-valued process defined above.

Define the function $\mathcal{H}:(\mathcal{P}(\mathcal{P}(\mathbb{I}))) \times(\mathcal{I})^{\otimes \mathbb{N}, *} \rightarrow[0,1]$ by

$$
\begin{aligned}
& \mathcal{H}(\nu, \mathcal{G})=\sum_{k=1}^{N_{1}} \prod_{i=1}^{N_{2, k}} \int\left[\mu_{i}^{\otimes n_{k i}}\left(A_{k, i}\right)\right] \nu\left(d \mu_{i}\right) \quad \text { if } \mathcal{G}=\bigcup_{k=1}^{N_{1}} \bigotimes_{2, i=1}^{N_{2, k}} A_{k, i}, \text { with } A_{k, i} \subset(\mathbb{I})^{n_{k i}}, \\
& \text { with } \bigotimes_{2, i=1}^{N_{2, k_{1}}} A_{k_{1}, i} \cap \bigotimes_{2, i=1}^{N_{2, k_{2}}} A_{k_{2}, i}=\varnothing \text { if } k_{1} \neq k_{2},
\end{aligned}
$$

we can also write this as $\mathcal{H}(\nu, \mathcal{G})=\nu^{*}(\mathcal{G})$. 
Theorem 3 Dual Representation

(a) For any solution $\left\{P_{\Xi_{0}}: \Xi_{0} \in \mathcal{P}(\mathcal{P}(\mathbb{I}))\right\}$ of the $\left(G^{N_{2}}, D_{2}\right)$ or $\left(G_{2}, D_{2}\right)$-martingale problem

$$
E_{\Xi_{0}}\left(\mathcal{H}\left(\Xi_{t}, \mathcal{G}_{0}^{2}\right)\right)=E_{\mathcal{G}_{0}}\left(\mathcal{H}\left(\Xi_{0}, \mathcal{G}_{t}^{2}\right)\right)
$$

(b) The $\left(G^{N_{2}}, D_{2}\right)$ and $\left(G_{2}, D_{2}\right)$-martingale problems are well-posed.

Proof The proof for the cases $\left(G^{N_{2}}, D_{2}\right)$ and $\left(G_{2}, D_{2}\right)$ follow the same lines. We now give the details the the latter case.

(a) As above, we begin by identifying the terms in $G_{2} H(\nu)$ for functions in $D_{2}$ of the form:

$$
H(\nu)=\prod_{k=1}^{k_{0}}\left(\int_{\mathcal{P}(\mathbb{I})}\left[\int_{\mathbb{I}^{n_{k}}} h\left(x_{k, 1}, \ldots, x_{k, n_{k}}\right) d \mu_{k}^{\otimes n_{k}}\right] \nu\left(d \mu_{k}\right)\right),
$$

in other words we work with functions of the form

$$
\prod_{k=1}^{k_{0}} h\left(x_{k, 1}, \ldots, x_{k, n_{k}}\right)
$$

The transitions for functions of this form are given as follows:

- Level I resampling. This results in the coalescence

$$
\int\left[\iint h\left(x_{11}, x_{12}\right) \mu\left(d x_{11}\right) \mu\left(d x_{12}\right)\right] \nu(d \mu) \rightarrow \int\left[\int h\left(x_{11}, x_{11}\right) \mu\left(d x_{11}\right)\right] \nu(d \mu), \quad \text { at rate } \gamma_{1}
$$

- Migration. At rate $c$

$$
\begin{aligned}
& \int\left[\int h\left(x_{11}, x_{12}\right) \mu\left(d x_{11}\right) \mu\left(d x_{12}\right)\right] \nu(d \mu) \\
& \rightarrow \iint\left[\int h\left(x_{11}, x_{22}\right) \mu_{1}\left(d x_{11}\right) \mu_{2}\left(d x_{22}\right)\right] \nu\left(d \mu_{1}\right) \nu\left(d \mu_{2}\right) .
\end{aligned}
$$

- Selection at level I with

$$
V_{1}(x)=s_{1} 1_{B}(x)
$$

This results in the transition with

$$
\begin{aligned}
& \iint h\left(x_{11}\right) \mu\left(d x_{11}\right) \nu(d \mu) \\
& \rightarrow \int\left[\int h\left(x_{11}\right) 1_{B}\left(x_{11}\right) \mu\left(d x_{11}\right)+\iint 1_{B^{c}}\left(x_{11}\right) h\left(x_{12}\right) \mu\left(d x_{11}\right) \mu\left(d x_{12}\right)\right] \nu(d \mu)
\end{aligned}
$$

at rate $s_{1}$. 
Under level II coalescence two occupied demes $i, j \in S, i \neq j$ are chosen at random at rate $\gamma_{2} / 2$ and we have

$$
\begin{gathered}
\int\left[\iint h\left(x_{i 1}, x_{i 2}\right) h\left(x_{j 1}, x_{j 2}\right) \mu_{i}\left(d x_{i 1}\right) \mu_{i}\left(d x_{i 2}\right) \mu_{j}\left(d x_{j 1}\right) \mu_{j}\left(d x_{j 2}\right)\right] \nu\left(d u_{i}\right) \nu\left(d \mu_{j}\right) \\
\rightarrow \int\left[\iiint \int h\left(x_{i 1}, x_{i 2}\right) h\left(x_{i 3}, x_{i 4}\right) \mu\left(d x_{i 1}\right) \mu\left(d x_{12}\right) \mu\left(d x_{i 3}\right) \mu\left(d x_{i 4}\right)\right] \nu(d \mu), \\
h \otimes_{2} h \rightarrow h \otimes h .
\end{gathered}
$$

In particular we have

$$
\int\left(\int h(x) \mu(d x)\right) \nu(d \mu) \cdot \int\left(\int h(x) \mu(d x)\right) \nu(d \mu) \rightarrow \int\left(\int h(x) \mu(d x)\right)^{2} \nu(d \mu) .
$$

Level II selection with fitness function $V_{2}$

Now consider the case in which $h($.$) and V_{2}(\cdot)$ are polynomials, that is,

$$
h(\mu)=\sum_{j} h_{j} \mu^{\otimes}\left(\otimes_{1, i} A_{j i}\right) \quad \text { polynomial on } \mathcal{P}(\mathbb{I}), h_{j} \leq 1
$$

where

$$
\begin{gathered}
V_{2}(\mu)=\sum_{j} a_{j} V_{2, j}, \quad V_{2, j}=\mu^{\otimes}\left(\otimes_{1, i} B_{j i}\right) \\
h(\mu) \rightarrow V_{2, j}\left(\mu_{1}\right) h\left(\mu_{1}\right)+\left(1-V_{2, j}\left(\mu_{1}\right)\right) h\left(\mu_{2}\right) \quad \text { at rate } a_{j} . \\
\int h(\mu) \nu(d \mu) \rightarrow \int V_{2, j}\left(\mu_{1}\right) h\left(\mu_{1}\right) \nu\left(d \mu_{1}\right)+\iint\left(1-V_{2, j}\left(\mu_{1}\right)\right) h\left(\mu_{2}\right) \nu\left(d \mu_{1}\right) \nu\left(d \mu_{2}\right) \\
h\left(x_{11}, \ldots, x_{1, n_{1}}\right) \rightarrow \\
V_{2}\left(x_{1,1}, \ldots, x_{1, n_{2}}\right) h\left(x_{\left(1, n_{2}+1\right)}, \ldots, x_{1,\left(n_{2}+n_{1}\right)}\right) \\
+\left(1-V_{2}\left(x_{1,1}, \ldots, x_{1, n_{2}}\right)\right) h\left(x_{2,\left(n_{2}+1\right)}, \ldots, x_{2,\left(n_{2}+n_{1}\right)}\right)
\end{gathered}
$$

More generally, if $H(\nu)=\int \prod_{i=1}^{K} h\left(\mu_{i}\right) \nu\left(d \mu_{i}\right)$ Assume $V_{2} \leq 1$, namely the indicator function of a set. The selection acting on $H$ produces

$$
\begin{aligned}
& \int \prod_{i=1}^{K} h\left(\mu_{i}\right) \nu\left(d \mu_{i}\right) \longrightarrow \\
& \sum_{j=1}^{K}\left(\left\{\prod_{i \neq j} h\left(\mu_{i}\right) \nu\left(d \mu_{i}\right)\right\}\right. \\
& \left.\cdot\left[\int h\left(\mu_{j}\right) V_{2}\left(\mu_{j}\right) \nu\left(d \mu_{j}\right)+\int\left(1-V_{2}\left(\mu_{j}\right)\right) \nu\left(d \mu_{j}\right) \int h\left(\mu_{K+1}\right) \nu\left(d \mu_{K+1}\right)\right]\right)
\end{aligned}
$$




$$
h\left(\mu_{1}\right) \rightarrow V_{2}\left(\mu_{1}\right) h\left(\mu_{1}\right)+\left(1-V_{2}\left(\mu_{1}\right)\right) \otimes h\left(\mu_{2}\right) .
$$

The corresponding set-valued transitions are obtained by restricting the class of functions $h$ of the form $h(\mu)=\mu^{\otimes}(A)$ with $A \in \mathcal{I}$ as in subsubsection 3.2 .

Coupling. In view of the assumption of exchangeability, we can couple the $V_{2}(\mu) h(\mu)$ and $\overline{\left(1-V_{2}(\mu)\right)}$ terms for the level II selection transitions at the deme level, that is, place these at the same deme index in the two resulting summands thus producing a union of disjoint sets in $\mathrm{I}^{2 *}$. Then as before, all operations are performed simultaneously on all demes and ranks in the different summands.

$\underline{\text { Set-valued transitions }}$ The set-valued transitions can then be read off by restricting the functionvalued transitions to the class of functions $h$ that are based on indicator functions of sets as in (3.61) and noting that due to the coupling the transitions preserve the decomposition into the union of disjoint subsets. These transitions define a Markov jump process $\left\{\mathcal{G}_{t}^{2}\right\}_{t \geq 0}$ with countable state space $\mathrm{I}^{2 *}$ and we denote the resulting generator by $G^{\text {dual }}$. The identity of the action of the corresponding terms of $G_{2}$ acting on $\mathcal{H}(\nu, \mathcal{G})$ and the result of the transition of the set-valued dual, that is,

$$
G_{2} \mathcal{H}(\nu, \mathcal{G})=G^{\text {dual }} \mathcal{H}(\nu, \mathcal{G}) \quad \text { for all } \nu \in \mathcal{P}(\mathcal{P}(\mathbb{I})), \mathcal{G} \in(\mathcal{I})^{\otimes \mathbb{N}, *}
$$

is then immediate by inspection. For example, setting $V_{2}(\mu)=\mu^{\otimes}\left(\otimes_{1, i} B_{i}\right)$ and applying the corresponding selection transition from $G_{2}$ to $H(\nu)=\int \prod_{i=1}^{K} h_{i}\left(\mu_{i}\right) \nu\left(d \mu_{i}\right)$ with $h_{i}\left(\mu_{i}\right)=$ $\mu_{i}\left(\otimes_{1, j} A_{i j}\right)$, 3.66) yields the set-valued transitions (3.51) with $A_{i}=\otimes_{1, j} A_{i j}$ which correspond the $G^{\text {dual }}$.

The duality relation (3.54) then follows from (3.67) (see for example Proposition 7.10 in [17] or Chapter 4 of [27])).

The uniqueness of the solution to the martingale problem is then obtained. In particular, moment measures of the time marginals of any solution to the martingale problem are determined by the dual representation. In turn the moment measures uniquely define the $\mathcal{L}(\Xi(t))$ as follows: Let $\widetilde{\mu}_{1}, \widetilde{\mu}_{2}, \ldots$ be an exchangeable sequence of $\mathcal{P}(\mathbb{I})$-valued random variables with marginal distributions given by the moment measures determined by the dual $\mathcal{G}_{t}^{2}$ and

$$
\widetilde{\Xi}_{m}:=\frac{1}{m} \sum_{i=1}^{m} \delta_{\widetilde{\mu}_{i}} .
$$

Then by de Finetti's theorem

$$
\mathcal{L}\left(\widetilde{\Xi}_{m}\right) \Rightarrow \mathcal{L}(\Xi(t)) \quad \text { as } m \rightarrow \infty,
$$

that is, the time marginal laws of any solution are uniquely determined by this limit.

(b) Since the class of function of the form $\mathcal{H}(\cdot, \mathcal{G})$ with $\mathcal{G} \in(\mathcal{I})^{\otimes \mathbb{N} \text {,* }}$ is probability-measuredetermining on $\mathcal{P}(\mathcal{P}(\mathbb{I})$ ) this implies that the time marginals of $\Xi(t)$ are uniquely determined 
follows from (a). The result (b) then follows from the basic results on dual martingale problems (see e.g. Theorem 7.9 and Proposition 7.10 in [17] or Chapter 4 of [27]).

\subsubsection{Moment calculations}

The dual can be used to compute joint moments and covariance structures. We illustrate with two simple examples.

Example 6 Consider the case of $\mathbb{I}=\{1,2\}$, no mutation and $V_{1}(1)=1, V_{1}(2)=0, c=\gamma_{2}=$ $s_{2}=0$ but $s_{1}, \gamma_{1}>0$. In order to compute

$$
\lim _{t \rightarrow \infty} E_{\delta_{\mu_{0}}}\left(\int \mu(2) \Xi_{t}(d \mu)\right)
$$

we use the the dual started with $\mathcal{G}_{0}=(01)$. Then we have transitions due to selection and coalescence. As a result

$$
\mathcal{G}_{t}=(01)^{\otimes_{1} n(t)}
$$

where $n(t)$ is a birth and death process with linear birth rate $n \rightarrow n+1$ at rate $s_{1} n$ and quadratic death rate $\gamma_{1} n(n-1) / 2$. As a result $\{n(t)\}$ is ergodic with distribution measure $\left\{p_{k}: k \in \mathbb{N}\right\}$. Then

$$
\lim _{t \rightarrow \infty} E_{\delta_{\mu_{0}}}\left(\int \mu(2) \Xi_{t}(d \mu)\right)=E\left(\mu_{0}^{\otimes 1}\left(\mathcal{G}_{e q}\right)\right)=\sum_{k=1^{\infty}}\left(\mu_{0}(2)\right)^{k} p_{k}
$$

Next consider

$$
\lim _{t \rightarrow \infty} E_{\delta_{\mu_{0}}}\left(\int(\mu(1) \cdot \mu(2)) \Xi_{t}(d \mu)\right) .
$$

In this case we start the dual with $\mathcal{G}_{0}=(10) \otimes_{1}(01)$. Again the number of ranks is given by a birth and death process which will eventually reduce to $n(t)=1$ which due to coalescence implies that $\mathcal{G}(\tau)=\emptyset$ for some finite random time $\tau$. This implies fixation, that is, the limit (3.73) is zero. The corresponding fixation probabilities are then given by the limiting first moments calculated in (3.79).

Example 7 With two types $\{1,2\}$ we take $\mathcal{G}_{0}^{2}=(10)_{1}^{\otimes k_{1}} \otimes_{2}(10)_{2}^{\otimes k_{2}}$ and we get

$$
E_{\Xi_{0}}\left(\int(\mu(1))^{k_{1}} \Xi_{t}(d \mu) \int(\mu(1))^{k_{2}} \Xi_{t}(d \mu)\right)=E\left[\mathcal{H}\left(\Xi_{t},(10)_{1}^{\otimes k_{1}} \otimes_{2}(10)_{2}^{\otimes k_{2}}\right)\right]=E_{\mathcal{G}_{0}^{2}}\left[\mathcal{H}\left(\Xi_{0}, \mathcal{G}_{t}^{2}\right)\right] .
$$

\subsubsection{Coalescent}

The coalescent plays a central role in the study of Moran, Fisher-Wright and Fleming-Viot processes with neutral types. We now consider the analogous genealogical structure for twolevel systems with $s_{1}=s_{2}=0$ which is determined by the level one and level two coalescence transitions in the set-valued dual. The genealogy is described by a marked coalescent process analogous to the marked coalescent process used for spatial processes (see for example [34]). 
The state space of the two-level coalescent is the set of marked partitions $(\pi, \zeta(\pi)), \pi \in$ $\Pi^{\mathrm{I}}, \zeta(\pi) \in \mathbb{N}^{|\pi|}$ where $\Pi^{\mathrm{I}}$ is the set of partitions of a countable set I into subsets and for $\pi \in \Pi^{\mathrm{I}}$, $|\pi|$ denotes the number of subsets in the partition. The marks $\{\zeta(i): i=1, \ldots,|\pi|\}$ represent the positions in $\mathbb{N}$ of the subsets and $|\zeta|=\mid\{k \in \mathbb{N}: \zeta(i)=k$ for some $i \in\{1, \ldots,|\pi|\}\} \mid$. For $i=1, \ldots,|\zeta|$, let $n_{i}(\pi)$ denote the number of subsets with $\zeta=i$ so that $\sum_{i=1}^{|\zeta(\pi)|} n_{i}(\pi)=|\pi|$.

A subset in the partition can jump to a new unoccupied site at rate $c$ and level I coalescence of two subsets occurs at rate $\gamma_{1}$ if they are at the same site. On the other hand all the subsets at two occupied sites combine to form a single site with all these subsets at rate $\gamma_{2}$.

Therefore given $\left(|\zeta(\pi)|,\left(n_{1}, \ldots, n_{|\zeta(\pi)|}\right)=\left(k,\left(n_{1}, \ldots, n_{k}\right)\right)\right.$ the possible transitions are:

1. $\left(k,\left(n_{1}, \ldots, n_{k}\right)\right) \rightarrow\left(k+1,\left(n_{1}, \ldots, n_{i}-1, \ldots, n_{k}, 1\right)\right)$ at rate $c n_{i} 1_{n_{i}>1}$,

2. $\left(k,\left(n_{1}, \ldots, n_{k}\right)\right) \rightarrow\left(k,\left(n_{1}, \ldots, n_{i}-1, \ldots, n_{k}\right)\right)$ at rate $\gamma_{1} n_{i}\left(n_{i}-1\right)$,

3. $\left(k,\left(n_{1}, \ldots, n_{i}, \ldots, n_{j}, \ldots, n_{k}\right)\right) \rightarrow\left(k-1,\left(n_{1}, \ldots, n_{i}+n_{j}, \ldots, n_{k}\right)\right)$ at rate $\gamma_{2} k(k-1)$.

Proposition 3.1 (The multilevel coalescent)

(a) Consider the case with $s_{1}=s_{2}=c=0, \gamma_{2}>0$ and $\gamma_{1}>0$. Then the two level coalescent converges to $(1,(1))$.

(b) If $c>0$ and $\gamma_{1}>0, \gamma_{2}=0$, then the coalescent process started at $\left(k_{0},\left(n_{1}, \ldots, n_{k_{0}}\right)\right)$ with $k_{0}<\infty$, converges to $(\widetilde{k},(1, \ldots, 1))$ for some random $\widetilde{k}$.

Proof (a) Due to jumps of type 2 at rate $\gamma_{2}$ the process will eventually reach an element of the type $\left(1,\left(n_{1}, \ldots, n_{i}+n_{j}, \ldots, n_{1}\right)\right)$. The due to jumps of type 1 the element $(1,(1))$ is then reached in a finite random time.

(b) If $\gamma_{2}=0$, the number of occupied sites is nondecreasing and at each site level I coalescence leads to a single element.

\section{Long-time behaviour of two type multilevel population sys- tems}

The class of two-level Fleming-Viot systems obtained by Theorem 1 with dual representation given by Theorem 3 describe a rich class of population systems. The evolution of their structure over different time scales depends on various parameters including mutation, levels I and II selection, migration, and demographic stochasticity rates and lead to different classes of behaviours.

In this section we consider the simplest case of a system with two types $\mathbb{I}=\{1,2\}$, no mutation, migration rate $c$, levels I and II selection rates $s_{1}, s_{2}$ and levels I and II resampling rates $\gamma_{1}, \gamma_{2}$. We first consider the deterministic case (i.e. infinite population case at both levels, $\left.\gamma_{1}=\gamma_{2}=0\right)$ and then in the random cases $\gamma_{1}$ and/or $\gamma_{2}>0$.

\subsection{Nonlinear measure-valued dynamics with $\gamma_{1}=\gamma_{2}=\mathbf{0}$}

Proposition 4.1 Consider the two level system in which $\gamma_{2}=0$ and $P\left(\Xi_{0}=\nu_{0}\right)=1, \nu_{0} \in$ $\mathcal{P}(\mathcal{P}(\mathbb{I}))$. Then the $\mathcal{P}(\mathcal{P}(\mathbb{I}))$-valued process $\Xi_{t}=\nu_{t}$ is deterministic. 
Proof This follows immediately from the dual representation since the variance of $\operatorname{Var}\left(\int \mathrm{h}(\mu) \Xi_{\mathrm{t}}(\mu)\right)=$ 0 for any $h \in \mathbb{H}$. This can be shown to be zero since all $E\left[\left(\int h(\mu) \Xi_{t}(\mu)\right)^{2}\right]$ is obtained by starting the dual with

$$
h\left(\mu_{1}\right) \otimes_{2} h\left(\mu_{2}\right)
$$

and since no coalescence occurs the two descendant sets evolve independently.

Now assume that the mutation, migration and genetic drift parameters are zero and

$$
V_{1}(x)=1_{D}(x), D \subset \mathbb{I},
$$

and level II fitness function of the form:

$$
V_{2}(\mu)=\mu(B) \text { with } B \subset \mathbb{I} .
$$

(This can be generalized to $V_{2}(\mu)=\mu^{\otimes}(B)$ with $B \subset(\mathbb{I})^{K}$ for some $K \in \mathbb{N}$ but we consider here the case $K=1$ to keep things simple.)

The transitions of the dual due to level I, respectively, level II, are given by

$$
\begin{gathered}
1_{C}\left(x_{11}\right) \rightarrow 1_{D \cap C}\left(x_{11}\right)+1_{D^{c}}\left(x_{11}\right) \otimes_{1} 1_{C}\left(x_{12}\right) \quad \text { at rate } s_{1}, \\
1_{C}\left(x_{11}\right) \rightarrow 1_{B}\left(x_{11}\right) \otimes_{1} 1_{C}\left(x_{12}\right)+1_{B^{c}}\left(x_{11}\right) \otimes_{2} 1_{C}\left(x_{21}\right) \quad \text { at rate } s_{2} .
\end{gathered}
$$

Theorem 4 Assume that $\mathbb{I}=\{1,2\}$ with fitness functions

$$
V_{1}(1)=0, V_{1}(2)=1
$$

and

$$
V_{2}(\mu)=\mu(1)
$$

selection rates $s_{1} \geq 0, s_{2} \geq 2$ and all other parameters equal to zero. Then as $t \rightarrow \infty$,

(a) If $s_{1}>0, s_{2}=0$, and $\nu_{0} \neq \delta_{(1,0)}$, then $\nu_{t} \rightarrow\left(1-\nu_{0}((1,0))\right) \delta_{(0,1)}$,

(b) If $s_{1}=0, s_{2}>0$, for some $x_{0} \in(0,1], \nu_{0}\left(\left\{\mu: \mu(1) \geq x_{0}\right\}\right)>0$, then $\nu_{t} \rightarrow \delta_{\left(p^{*}, 1-p^{*}\right)}$ with $p^{*} \geq \int \mu(1) \nu_{0}(d \mu)$.

Proof We compute $\frac{d}{d t} E_{\nu_{0}}\left(\int \mu(1) \nu_{t}(d \mu)\right)$ using the equivalent dual form $\frac{d}{d t} E_{\mathcal{G}_{0}=\{1\}}\left(\mathcal{H}\left(\nu_{0}, \mathcal{G}_{t}\right)\right)$. To compute the latter consider the first dual transition, either a level I selection jump (4.76) or a level II selection jump (4.77). This yields $1_{1} \rightarrow 1_{1} \otimes_{1} 1_{1}$ at rate $s_{1}$ and $1_{1} \rightarrow 1_{1} \otimes_{1} 1_{1}+1_{1} \otimes_{2} 1_{2}$ at rate $s_{2}$ and therefore

$$
\frac{d}{d t} E_{t}(\mu(1))=s_{2} \operatorname{Var}_{2, t}(\mu(1))-s_{1}\left(E_{t}(\mu(1))-\left(E_{t}(\mu(1))\right)^{2}\right)
$$

where

$$
E_{t}(\mu(1))=\int \mu(1) \nu_{t}(d \mu), \operatorname{Var}_{2, t}(\mu(1))=\int \mu(1)^{2} \nu_{t}(d \mu)-\left(\int \mu(1) \nu_{t}(d \mu)\right)^{2}
$$


(a) If $s_{2}=0$, then (4.80) yields the ode

$$
\frac{d}{d t} E_{t}(\mu(1))=s_{1}\left[\left(E_{t}(\mu(1))\right)^{2}-E_{t}(\mu(1))\right]
$$

and the result follows immediately.

(b) If $s_{1}=0$, then by (4.80) $E_{t}(\mu(1))$ is nondecreasing and strictly increasing if $\operatorname{Var}_{2, t}(\mu(1))>0$. Since $E(\mu(1)) \leq 1$ and $\operatorname{Var}_{2, t}(\mu(1))>0$ unless $\nu=\delta_{(x, 1-x)}$ for some $x \in[0,1], \lim _{t \rightarrow \infty} \operatorname{Var}_{2, t}(\mu(1))=$ 0 and $\nu_{t} \rightarrow \delta_{\left(p^{*}, 1-p^{*}\right)}$ with $p^{*} \geq \int \mu(1) \nu_{0}(d \mu)$.

In the case $\delta_{1}=0, \delta_{2}=0$, the system is deterministic. In order for level II selection to play a role, diversity in the composition of clusters is required as first pointed out by Maynard Smith (recall discussion in subsubsection 1.1.1). In particular consider the case where $\nu_{0} \in \mathcal{P}(\mathcal{P}(\mathbb{I})$ ), let $\widetilde{\nu}_{0}(d x):=\nu_{0}(\{\mu: \mu(1) \in d x\}) \in \mathcal{P}([0,1])$. The following is a version of a result of Luo [51].

Theorem 5 Assume (4.78) and (4.79), $c=0$, and $s_{1}>0$. Assume that $\widetilde{\nu}_{0}(d x)$ has a continuous density $\widetilde{\nu}_{0}(x)$. Then

(a) If there exists $x_{0}<1$ such that $\widetilde{\nu}_{0}\left(\left[x_{0}, 1\right]\right)=0$, then $\widetilde{\nu}_{t} \rightarrow \delta_{0}, \nu_{t} \rightarrow \delta_{(0,1)}$.

(b) If the density $\widetilde{\nu}_{0}$ is continuous and positive at 1 , then there exists a critical s. such that if $s_{2}<s_{2}^{*}$, then $\nu_{t} \rightarrow \delta_{(0,1)}$ and if $s_{2}>s_{2}^{*}$, then there exists an equilibrium distribution $\nu_{e q}$ with $\nu_{e q}(\{\mu: \mu(1)>0\})>0$.

Proof This was established by S. Luo [51] (see (A.5) in the case $\eta=U$ ) by solving an integro partial differential equation for $\widetilde{\nu}_{t}(x)$, the density of the distribution of $\mu_{t}(1)$. Luo's equation is given by

$$
\frac{\partial}{\partial t} \widetilde{\nu}(t, x)=\frac{\partial}{\partial x}(\widetilde{\nu}(t, x) x(1-x))+\lambda \widetilde{\nu}(t, x)\left[x-\int_{0}^{t} \widetilde{\nu}(t, y) y d y\right]
$$

with solution

$$
\widetilde{\nu}(t, x)=\widetilde{\nu}_{0}\left(\frac{x e^{t}}{1+x\left(e^{t}-1\right)}\right) e^{t-\lambda \int_{0}^{t} h(s) d s}\left[1+x\left(e^{t}-1\right)\right]^{(\lambda-2)}
$$

where $\lambda=\frac{s_{2}}{s_{1}}$ and $h(t)=\int_{0}^{1} y \widetilde{\nu}(t, y) d y$.

(a) For any $\varepsilon>0$ there exists $t_{0}(\varepsilon)$ such that for $x \geq \varepsilon$ and $t \geq t_{0}(\varepsilon), \frac{x e^{t}}{1+x\left(e^{t}-1\right)} \geq x_{0}$ and therefore the right side of (4.83) is 0 .

(b) In the case $\mu_{0}$ is uniform on $[0,1]$ and $\lambda \neq 1$, 44.83 becomes

$$
\widetilde{\nu}(t, x)=\frac{\left(e^{t}-1\right)(\lambda-1)}{e^{t(\lambda-1)}-1}\left[1+x\left(e^{t}-1\right)\right]^{(\lambda-2)}
$$

where $\lambda=s_{2} / s_{1}$. When $\lambda<1$, we have

$$
\int_{0}^{1} x \widetilde{\nu}(t, d x) \rightarrow 0 .
$$

When $\lambda>1$, we get

$$
\widetilde{\nu}_{e q}(1)=\lim _{t \rightarrow \infty} \widetilde{\nu}(t, 1) \rightarrow \lambda-1,
$$


and

$$
\int_{0}^{1} x \widetilde{\nu}(t, d x) \rightarrow \frac{\lambda-1}{\lambda}
$$

From Theorem 5(a) it follows that the survival of type 1 depends only on the initial density $\widetilde{\nu}(0, x)$ near $x=1$ and (4.86) remains positive if the initial density is positive at $x=1$. This means that level II selection can overcome level I selection with two types only if there is a positive density of sites (subpopulations) at time $t=0$ with arbitrarily small (or zero) proportions of the individually more fit types. We next consider the role of randomness and demonstrate that level one positive genetic drift can lead to a phase transition with the possibility of survival of inferior types for any initial $\nu_{0}$ provided that $\int \mu(1) \nu_{0}(d \mu) \in(0,1)$.

\subsection{Phase transitions: the role of randomness}

In this subsection we again consider the case in which level two selection favours colonies that include altruistic or cooperative types. In the previous subsection level II selection exploited the diversity in the initial distribution among demes.

\subsubsection{Level I randomness}

In this subsection we now consider the parameter regions for dominance of level I or level II selective effects and the transition between these phases where level II selection acts on the diversity in deme composition resulting from local genetic drift at each deme, that is, when $\gamma_{1}>0$.

Theorem 6 Consider the case $\mathbb{I}=\{1,2\}$. Assume that, $c>0, \gamma_{1}>0, \gamma_{2}=0, s_{1} \geq 0, s_{2} \geq 0$, with $\int \mu(1) \nu_{0}(d \mu) \in(0,1)$.

$$
V_{1}(1)=0, V_{1}(2)=s_{1}
$$

The migration rate is $c$ and the deme fitness is

$$
V_{2}(\mu)=s_{2} \mu(1)
$$

(a) Assume $m_{12}=m_{21}=0$ and $s_{2}=0, s_{1}>0$. Then $\int_{\mathcal{P}(\mathbb{I})} \mu(2) \nu(d \mu) \rightarrow 0$ with exponential decay rate.

(b) Assume $m_{12}=m_{21}=0$ and $s_{1}=0, s_{2}>0$. Then $\int_{\mathcal{P}(\mathbb{I})} \mu(1) \nu(d \mu) \rightarrow 0$ with exponential decay rate.

(c) Assume $m_{12}=m_{21}=0$. Then for fixed $c>0, \gamma_{1}>0, s_{1}>0$, there is a critical value $s_{2}^{*}\left(c, \gamma, s_{1}\right) \in(0, \infty)$ such that level II selection dominates, that is then for $\varepsilon>0$,

$$
\nu_{t}(\{\mu: \mu(2)>\varepsilon\}) \rightarrow 0
$$


if $s_{2}>s_{2}^{*}$ and level I selection dominates if $s_{2}<s_{2}^{*}$, that is, for $\varepsilon>0$,

$$
\nu_{t}(\{\mu: \mu(1)>\varepsilon\}) \rightarrow 0 .
$$

(d) Assume that $m_{12}, m_{21}>0$. The there exists a unique equilibrium and the system converges to the equilibrium measure as $t \rightarrow \infty$.

Proof (a) and (b). We use the dual $\left\{\mathcal{G}_{t}\right\}$ with initial value $\mathcal{G}_{0}=\{2\}$ ( or $\{1\}$ ) to compute the first moment of $E_{\Xi_{0}}\left[\int \mu(2) \Xi_{t}(d \mu)\right]=E_{\mathcal{G}_{0}}\left[H\left(\Xi_{0}, \mathcal{G}_{t}\right)\right]$ where $\Xi_{0}=\delta_{\mu_{0}}$. Let $p=\mu_{0}(2)$.

We now identify two basic combinations of transitions that will either increase or decrease this expression.

(1) Level I selection followed by migration before coalescence.

Transitions in terms of indicator functions of the sets,

$$
\begin{aligned}
1_{2}(1) & \rightarrow 1_{2}(1)+1_{1}(1) \otimes_{1} 1_{2}(1) \quad \text { level I selection at rate } s_{1} \\
& \rightarrow 1_{2}(1)+1_{1}(1) \otimes_{2} 1_{2}(2) \text { migration }
\end{aligned}
$$

so that after integration we obtain

$$
p \rightarrow p+(1-p) p=2 p-p^{2}=1-(1-p)^{2} .
$$

Note that after the migration step, this change can no longer be reversed by a coalescence at site 1 . Noting that migration before coalescence occurs with probability $\frac{2 c}{2 c+\gamma_{1}}$ so that this combination occurs with effective rate $s_{1} \frac{2 c}{2 c+\gamma_{1}}$.

Similarly these transition result in

$$
\begin{aligned}
1_{1}(1) & \rightarrow 0+1_{1}(1) \otimes_{1} 1_{1}(1) \quad \text { level I selection } \\
& \rightarrow 1_{1}(1) \otimes_{2} 1_{1}(2) \text { migration } \\
(1-p) & \rightarrow(1-p)^{2}
\end{aligned}
$$

In the absence of level II selection this can be identified with a Crump-Mode-Jagers (CMJ) branching process (see [17](3.1.4), [57] ) in which individuals are occupied sites and during their lifetimes these individuals produce new offspring sites. Since the death rate is zero this is a supercritical branching process with exponential growth rate, the Malthusian parameter $\alpha_{1}$ (see 24] subsubsection 8.3.4) which can be represented in terms of the stable age distribution [24],(8.167). This proves (a).

(2) Level II selection. We consider the effect of a level II selection event. followed by coalescence before migration.

$$
\begin{aligned}
1_{2}(1) \quad \begin{array}{l}
\rightarrow \\
1_{1}(1) \otimes_{1} 1_{2}(1)+1_{2}(1) \otimes_{2} 1_{2}(2) \quad \text { level II selection at rate } s_{2}
\end{array} \\
\rightarrow 1_{1}(1) \otimes_{2} 1_{2}(2)+1_{2}(1) \otimes_{2} 1_{2}(2)=1_{2}(2) \\
\quad \text { migration before coalescence with probability } \frac{2 c}{2 c+\gamma_{1}} \\
\rightarrow 0+1_{2}(1) \otimes_{2} 1_{2}(2) \quad \text { coalescence before migration with probability } \frac{\gamma_{1}}{2 c+\gamma_{1}}
\end{aligned}
$$


so that after integration we have

$$
p \rightarrow p, \quad p \rightarrow p^{2},
$$

in the two cases.

Similarly in the second case (coalescence before migration) we have

$$
\begin{aligned}
1_{1}(1) & \rightarrow 1_{1}(1) \otimes_{1} 1_{1}(1)+1_{2}(1) \otimes_{2} 1_{1}(2) \quad \text { level II selection } \\
& \rightarrow 1_{1}(1)+1_{2}(1) \otimes_{2} 1_{1}(2) \quad \text { coalescence before migration with probability } \frac{\gamma_{1}}{2 c+\gamma_{1}},
\end{aligned}
$$

so that after integration we have

$$
(1-p) \rightarrow(1-p)+p(1-p)=1-p^{2}
$$

and this occurs with effective rate $s_{2} \frac{\gamma_{1}}{2 c+\gamma_{1}}$. In the absence of level I selection this again produces a supercritical CMJ branching process with Malthusian parameter $\alpha_{2}$ in which individuals are occupied sites (where an occupied site is a site at which the set is not $\mathbb{I}$ ). This proves (b).

(c) When both $s_{1}>0, s_{2}>0$ there is a competition between the two levels of selection. In the dual setting this corresponds to the the competition between two branching mechanisms, that is, competing CMJ branching processes.

We now focus on the interaction of the competing CMJ processes. To do this we first consider effect of a level I transition at a site where a level II transition has already occurred. This leads to

$$
1_{2} \rightarrow_{I I} 1_{2} \otimes_{2} 1_{2} \rightarrow_{I} 1_{2} \otimes_{2} 1_{2}+1_{2} \otimes_{2} 1_{1} \otimes_{2} 1_{2}
$$

that is, in terms of sets

$$
\{2\} \supset\left(\{2\} \otimes_{2}\{2\}\right) \subset\left(\{2\} \otimes_{2}\{2\}\right) \cup\left(\{2\} \otimes_{2}\{1\} \otimes_{2}\{2\}\right) \subset\{2\}
$$

which after integration leads to

$$
p \rightarrow_{I I} p^{2} \rightarrow_{I} p^{2}+p^{2}(1-p) \in\left[p, p^{2}\right] .
$$

This means that a level I transition after a level II transition partially reverses the first effect (but does not overshoot the reversal). (The same happens if a level II follows a level I transition.) Therefore we can obtain a bound to the decrease in the mean of type 2 due to level II transitions by completely reversing them at the rate of level I transitions.

First, assume that $s_{1} \frac{c}{c+\gamma_{1}}>s_{2} \frac{\gamma_{1}}{\gamma_{1}+c}$. We can construct a birth and death process with births (new factors $1_{1}(\cdot)$ via level I selection) at rate $s_{1} \frac{c}{c+\gamma_{1}}$. We note that we can obtain a domination by letting the action of level II selection to remove a $1_{1}(\cdot)$ (i.e. replacing it by $1_{2 \cup 1}$ rather than something intermediate), that is this produces a death rate $s_{2} \frac{\gamma_{1}}{c+\gamma_{1}}$. Therefore the resulting birth and death process is supercritical and goes to infinity. Thus in this case, if $\int \mu(2) \nu_{0}(d \mu)>0$, then $\int \mu(2) \nu_{t}(d \mu) \rightarrow 1$ and type 2 takes over in the limit in the McKean-Vlasov system. 
Similarly, if $s_{2} \frac{c}{c+\gamma_{1}}>s_{1} \frac{\gamma_{1}}{\gamma_{1}+c}$ then we have a birth process with $1_{2}$ factors produced due to level II selection at rate $s_{2} \frac{\gamma_{1}}{c+\gamma_{1}}$ and a removal of these factors by level I selection at rate $s_{1} \frac{\gamma_{1}}{c+\gamma_{1}}$. Therefore if $\int \mu(1) \nu_{0}(d \mu)>0$, then $\int \mu(1) \nu_{t}(d \mu) \rightarrow 1$ and type 1 wins out and takes over in the McKean-Vlasov system.

(d) This is a special case of Theorem 8 .

Remark 6 We can also consider the linear stability of the fixed points $\delta_{(1,0)}, \delta_{(0.1)}$ by computing

$$
\left.\frac{d}{d t} E_{(\varepsilon, 1-\varepsilon)}(\mu(1))\right|_{t=0}=s_{2} \gamma_{1}-s_{1} c
$$

for small $\varepsilon$ (e.g. under the condition $s_{2} \leq c$ discussed below). This is given by the first change due to a jump of the dual process started at $1_{1}$. This means that level II selection prevails if

$$
s_{2} \gamma_{1}>s_{1} c \text {. }
$$

Condition (4.93) was derived by Kimura 49 using the properties of the equilibria of the solution of equation 1.1 for the density $U(t, x)$. Existence and uniqueness of the solution to (1.1) was established by Shimakura [75]), namely, for any initial distribution there exists a unique solution. Also see Shiga 77] for a generalization to the multitype case. A more complete description of the long-time behaviour is proved in Ogura and Shimakura [61] Theorem 3(iii) (in the case $s_{2} \leq c$ so that $s_{1} / \gamma_{1} \leq 1$ if also $s_{2}>s_{1} c / \gamma_{1}$ which corresponds to the case in which the expected number of dual factors at a site is bounded above by 2). They also obtain a related result in Theorem 5 in the case in which the mutation rates satisfy $m_{12}>0, m_{21}>0$ or $m_{12}>0, m_{21}=0$ or $m_{12}=0, m_{21}>0$. These results were obtained using ODE methods and explicit solutions in terms of hypergeometric functions.

The ODE methods cannot be extended to populations having more than two types or systems with level II coalescence. The objective of this paper is to develop the dual representation and the above model of competing branching mechanisms to study the long-time behaviour of multitypemultilevel systems. An example involving three types is given in Theorem 10 .

\subsubsection{The case $\gamma_{1}>0, c=0$}

Consider the case again with no mutation, level II fitness function $V_{2}(\mu)=\mu(2), s_{2}>0$, $c=\gamma_{2}=0$ but with $\nu_{0}=\delta_{\mu_{0}}$, that is, no initial diversity in the composition of demes but with $\mu_{0}(i)>0 . i=1,2$. Then level II selection can dominate, that is, $\mu_{t}(2) \rightarrow 1$ only if $\gamma_{1}>0$. This means that group selection cannot be effective if $\gamma_{1}=0$ in the case $\nu_{0}=\delta_{\mu_{0}}$.

In the case $c=0, \gamma_{2}=0, \gamma_{1}>0$, fixation occurs within each deme so that eventually the competition is between demes of type 1 and demes of type 2 and then $\mu_{t}(2) \rightarrow 1$ if $s_{2}>0$. To verify this we have

$$
\begin{aligned}
(10) & \rightarrow(01) \otimes_{1}(10)+(10) \otimes_{2}(10) \quad \text { at rate } s_{2} \\
& \rightarrow(10) \otimes_{2}(10) \quad \text { at rate } \gamma_{1}
\end{aligned}
$$




$$
\begin{aligned}
(10) & \rightarrow(10)+(01) \otimes_{1}(10) \quad \text { at rate } s_{1} \\
& \rightarrow(10) \quad \text { at rate } \gamma_{1} .
\end{aligned}
$$

Recalling that the coalescence dominates level I selection here due to the quadratic rate then effectively the dual develops as $(10)^{\otimes_{2} k(t)}$ and $k(t) \rightarrow \infty$. Then if $\mu_{0}(1)<1, \nu_{t}=\delta_{\mu_{t}}$ and $\mu_{t}(1) \rightarrow 0$ as $t \rightarrow \infty$.

\subsubsection{Level II randomness $\gamma_{2}>0$}

Proposition 4.2 (a) If $\gamma_{1}=0, \mu_{0}(2)<1 . \nu_{0}$-a.s., and $s_{1}>0, \gamma_{2}>0, c>0$, then

$$
E\left[\int \mu(2) \Xi_{t}(d \mu)\right] \rightarrow 0 \text { as } t \rightarrow \infty
$$

(b) If $\gamma_{1}>0$ and $\gamma_{2}>0$, then fixation occurs, that is,

$$
E\left(\int \mu(1) \Xi_{t}(d \mu) \cdot \int \mu(2) \Xi_{t}(d \mu)\right) \rightarrow 0, \quad \text { as } t \rightarrow \infty .
$$

Proof (a) This follows by a simple dual calculation as follows:

$$
\begin{gathered}
(01) \rightarrow(01) \otimes_{1}(01) \text { as rate } s_{1}, \\
(01) \rightarrow(01) \otimes_{1}(01) \rightarrow(01) \otimes_{2}(01) \text { at rate } c,
\end{gathered}
$$

but then

$$
(01) \otimes_{2}(01) \rightarrow(01) \otimes_{1}(01) \text { at rate } \gamma_{2}
$$

and

$$
\begin{aligned}
(01) & \rightarrow(01) \otimes_{1}(01)+(10) \otimes_{2}(01) \text { at rate } s_{2} \\
& \rightarrow(01) \otimes_{1}(01)+(10) \otimes_{1}(01)=(01) \text { at rate } \gamma_{2}
\end{aligned}
$$

Recalling that the coalescence dominates level II selection here due to the quadratic rate then effectively the dual develops as $(01)^{\otimes_{1} k(t)}$ and $k(t) \rightarrow \infty$. This implies that level II selection has no long term effect and the level I selection leads to $\int(\mu(2))^{k(t)} \nu_{0}(d \mu) \rightarrow 0$, as $t \rightarrow \infty$.

(b) This follows by a two-level version of the calculation in Example 6 . 


\section{Multitype multilevel population systems}

In this section we consider the evolution of more complex population systems with possibly many types, multilevel structure and different combinations of the basic mechanisms. In this case it is no longer possible to use the methods of one dimensional nonlinear dynamics or one dimensional diffusions, that is, methods involving ordinary differential equations. We will outline the application of the dual representations in some examples in this class.

\subsection{Equilibria and fixation probabilities}

An important application of the dual is to obtain results on ergodic properties of multilevel systems. We begin by reviewing the results for the case with only level I selection in 24].

Theorem 7 Ergodicity of McKean-Vlasov systems with level I selection. Assume that $\gamma_{2}=0$ and that at time $t=0$, all demes have the same individual distribution, say $\mu_{0}$, that is $\nu_{0}=\delta_{\mu_{0}}$. If the mutation rates are positive on $\mathbb{I}$ and positive migration rate $c>0$, then the limiting empirical process is given by a deterministic McKean-Vlasov dynamics $\left\{\nu_{t}\right\}_{t \geq 0}$ where $\nu_{t}=\mathcal{L}\left(\mu_{t}\right)$. Moreover, as $t \rightarrow \infty, \nu_{t}$ converges to a unique equilibrium $\nu_{e q}$.

Proof See [24] Theorem 12, Theorem 14 for the case $s_{2}=0$. The extension to include the case $s_{2}>0$ follows along the same lines.

We now consider the extension of the ergodicity result to the case in which $\gamma_{2}>0$.

Theorem 8 Ergodicity of two level Fleming-Viot systems. Assume that $\gamma_{2}>0$ and $s_{2} \geq 0$ and positive mutation rates on $\mathbb{I}$. Then the law of the two level Fleming-Viot process converges to a unique equilibrium $P_{\text {eq }} \in \mathcal{P}(\mathcal{P}(\mathcal{P}(\mathbb{I}))$ ).

Proof We adapt the proof of [24]. Due to level II coalescence the number of sites occupied by the dual can be reduced to 1 and this event is recurrent. Note that the equilibrium mean measure of a subset of $\mathbb{I}$ is given by the probability that the dual process starting at the indicator of the subset hits the absorbing point $\mathbb{I}^{\mathbb{N}}$ but this occurs with positive probability at each time the event occurs. Therefore an absorbing point is reached with probability 1 .

We now consider the case in which the mutation Markov chain has two or more ergodic classes. In this case the system can be non-ergodic. If $\gamma_{1}>0$, then eventually the population will be concentrated on one of the ergodic classes and the problem is to compute the fixation probabilities.

Theorem 9 Fixation probabilities

(a) Single deme. Assume that the initial configuration is iid $\mu_{0}$ and that the mutation chain has two or more ergodic classes $\mathbb{I}_{1}, \ldots, \mathbb{I}_{\ell}, \gamma_{1}>0$, and $s_{1} \geq 0$. Then the population is ultimately fixed on one of the classes and the probability that it is in class $k$ is given by the equilibrium measure of the dual chain started with $\mathcal{G}_{0}=\mathbb{I}_{k}$ integrated with respect to the initial measure $\mu_{0}$. 
(b) Two level Fleming-Viot systems. Assume that $\gamma_{1}>0, \gamma_{2}>0, V_{1}, V_{2}>0$ and that the mutation Markov chain has two or more ergodic classes. Then there is ultimate fixation of a single ergodic class and the law of the two level system converges to a random mixture of pure equilibrium single class populations.

Proof (a) We again use the dual representation. To verify ultimate fixation take $\mathcal{G}_{0}=\prod_{i=1}^{\ell^{\prime}} \mathbb{I}_{i} \subset$ $\mathbb{I}^{\ell}, 2 \leq \ell^{\prime} \leq \ell$. Then due to the quadratic rate coalescence, with probability $1, \mathcal{G}_{\tau}=\emptyset$ at some random time $\tau$ and this is a trap so that $\mathcal{G}_{t}=\emptyset$ for large $t$. To compute the probability that ergodic class $k$ is chosen, start the dual with $\mathcal{G}_{0}=\mathbb{I}_{k}$. The dual process jumps are within class mutation jumps, selection jumps and coalescence jumps. Due to coalescence the state $\mathcal{G}=\mathbb{I}_{k}$ is recurrent and therefore the dual is positive recurrent and converges to equilibrium as $t \rightarrow \infty$. The fixation probability (limiting probability that ergodic class $k$ is chosen) is then obtained by integrating with respect to the initial measure $\mu_{0}^{\otimes}$.

(b) We again use the dual process. Due to level II coalescence there will eventually be an equilibrium distribution of the number of sites occupied by the dual and in fact the single site situation occurs infinitely often. Then each time there is non-zero probability that one of the classes will be eliminated and the within class mutation will hit an absorbing point as in (a).

Example 8 Assume that $\mathbb{I}=\{1,2,3\}$, no mutation, $\gamma_{1}>0, \gamma_{2}>0$ and $\nu_{0}=\frac{1}{3} \delta_{\delta_{1}}+\frac{1}{3} \delta_{\delta_{2}}+\frac{1}{3} \delta_{\delta_{3}}$. We use the set-valued dual $\mathcal{G}_{t}$. If $\mathcal{G}_{0}=\{i\}_{1} \otimes_{2}\{j\}_{2}$, then by level II coalescence followed by level I coalescence, $\mathcal{G}_{\tau}=\emptyset$ at some finite random time $\tau$ if $i \neq j$ and therefore only one type survives. We also note that the dual process $\mathcal{G}_{t}$ starting from $\mathcal{G}_{0}=\{i\}_{1}$ is positive recurrent with $\{i\}_{1}$ as a renewal point - this is due to the levels I and II quadratic rate coalescence events (in contrast to the linear birth rates due to selection events).

Then "level II fixation" occurs, namely,

$$
\nu_{t} \rightarrow \delta_{\delta_{i}} \quad \text { with probability } p_{i}
$$

and the fixation probabilities $p_{i}$ are obtained by integrating the equilibrium dual $\mathcal{G}_{\text {eq }}(i)$ starting from $\mathcal{G}_{0}=\{i\}_{1}$, that is,

$$
p_{i}=\nu_{0}^{\otimes}\left(\mathcal{G}_{\mathrm{eq}}(i)\right) .
$$

\subsection{Examples of multilevel effects}

The class of multitype multilevel population systems with mutation and selection is extremely rich and can exhibit many complex dynamical behaviors. We do not discuss this in detail but now given an illustrative example of a simple effect of this type

\subsubsection{A model of cooperation.}

Consider the 3 type case $\mathbb{I}=\{1,2,3\}$ with level I fitness function

$$
V_{1}(1)=v_{1}, \quad V_{1}(2)=V_{1}(3)=0
$$


and level II fitness function

$$
V_{2}(\mu)=v_{2} \mu(2) \mu(3)
$$

with $v_{1}, v_{2}>0$. This models a cooperative interaction of two types 2 and 3 that endow a deme containing them with a positive advantage.

We consider the emergence of demes having population distributions $\left(p_{1}, p_{2}, p_{3}\right)$ when $\gamma_{2}=0$ satisfying

$$
v_{1} p_{1}<v_{2} p_{2} p_{3}
$$

Theorem 10 Assume that $c>0, \gamma_{1}>0$, rare mutation from type 1 to types 2,3 occurs at rates $m_{12}=m_{13}=\varepsilon>0$ and $m_{23}, m_{32}>0, m_{21}=m_{31}=0$.

(a) If $v_{2}=0$, then for sufficiently large $v_{1}>0$ we can have long time survival of type 1 , that is an equilibrium with positive mean proportion of type 1.

(b) There exists a critical value $v_{2}^{*}$ at which emergence of demes having threshold values satisfying (5.109) occurs for $v_{2}>v_{2}^{*}$, type 1 becomes extinct and types 2,3 go to equilibrium.

Proof (a) This has been proved for the case of two types in [24], Corollary 10.1. The proof of (a) follows along the same lines and elements of it are used in the proof of (b). We will outline the main steps. We use the dual started with $\mathcal{G}_{0}=\{1\} \otimes \mathbb{I}^{\otimes \mathbb{N}}$. The corresponding indicator function representation starts with $(100) \otimes(111)^{\otimes \mathbb{N}}$. Then selection followed by migration leads to new summands. Mutation has the effect of eliminating summands so that together we have a birth and death process. There are two possible outcomes. First the (100) can be a recurrent point and eventually will eventually change to (000) with probability 1 . The second possibility is that the birth and death process is supercritical and in this case the type 1 is positive for the invariant measure.

(b) We consider $\lim _{t \rightarrow \infty} \int \mu(2 \cup 3) \nu_{t}(d \mu)$ and $\lim _{t \rightarrow \infty} \int \mu(1) \nu_{t}(d \mu)$. Note that (using indicator functions of subsets of $\mathbb{I}$ ) the level II selection transitions gives

$$
\begin{aligned}
& (011) \rightarrow \\
& (010) \otimes_{1}(001) \otimes_{1}(011) \\
& +\left[(100) \otimes_{1}(111)+(010) \otimes_{1}(110)+(001) \otimes_{1}(111)\right] \otimes_{2}(011)
\end{aligned}
$$

In order to produce a permanent summand we then require either two migration or mutation transitions before coalescence of the first two terms but this occurs with positive rate so that this can have an important effect for large $v_{2}$. This can then lead to

$$
\begin{aligned}
& (011) \rightarrow \\
& (010) \otimes_{1}(001) \otimes_{1}(011) \\
& +\left[(100) \otimes_{1}(111)+(010) \otimes_{1}(110)+(001) \otimes_{1}(111)\right] \otimes_{2}(011) \\
& \ldots \rightarrow(011)+(100) \otimes_{2}(011)
\end{aligned}
$$

Level I selection leads to

$$
(011) \rightarrow(011) \otimes_{1}(011)
$$


If we then have a level II selection

$$
\begin{aligned}
& (011) \rightarrow(011) \otimes_{1}(011) \\
& \rightarrow(010) \otimes_{1}(001) \otimes_{1}(011) \otimes_{1}(011) \\
& +\left[(100) \otimes_{1}(111)+(010) \otimes_{1}(110)+(001) \otimes_{1}(111)\right] \otimes_{2}(011) \otimes_{1}(011) \\
& \rightarrow \ldots(011) \otimes_{1}(011)+(011) \otimes_{1}(100) \otimes_{2}(011)
\end{aligned}
$$

We then have competing branching mechanisms one whose rate is proportional to $v_{1}$ and one whose rate is proportional to $v_{2}$. We can construct birth and death processes where the deaths in the level I process (birth rate prop. to $v_{1}$ ) are caused by mutation and level II selection. Deaths in the level II process (birth rate prop to $v_{2}$ )are caused by level I selection. Recall that type 1 can only survive if the level birth and death process is supercritical (cf. DG, p. 743). We again have a dichotomy involving the critical behaviour of two competing branching processes and the result follows as in the proof of Theorem 6

\subsubsection{Emergence of mutualistic types}

There are many different mechanisms involving multilevel selection that can influence the overall population structure. For example, multilevel selection can make possible the survival of a trait that then leads to the emergence of another trait that has a mutually beneficial effect which then gives the first trait higher (inclusive) individual fitness and the pair survives locally.

To illustrate this consider the following modification of the model of subsubsection 5.2.1. Let

$$
V_{1}(1)=v_{1}, \quad V_{1}(2, \mu)=v_{M} \cdot \mu(3), V_{1}(3, \mu)=v_{M} \cdot \mu(2),
$$

and level II fitness function

$$
V_{2}(\mu)=v_{2}(\mu(2)+\mu(3))
$$

Theorem 11 Assume that $c \geq 0, \gamma_{1} \geq 0$, rare mutation from type 1 to types 2,3 occurs at rates $m_{12}=m_{13}=\varepsilon \geq 0$ very small, and with $v_{1}, v_{2}, v_{M} \geq 0$. Denote by $x_{i}(t)$ the expected proportion of type $i$ at time $t$.

(a) Consider the deterministic case with $\gamma_{1}=m_{12}=m_{13}=0, v_{1}=1$ and $v_{2}=0$ and for simplicity assume that $x_{2}(0)=x_{3}(0)>0$. Then if

$$
v_{M}<\frac{2}{1-x_{1}(0)}
$$

then

$$
x_{1}(t) \rightarrow 1
$$

as $t \rightarrow \infty$.

(b) If

$$
v_{M}>\frac{2}{1-x_{1}(0)},
$$


then $x_{1}(t) \rightarrow 0$ as $t \rightarrow \infty$. In the case $v_{M}=\frac{2}{1-x_{1}(0)}$ there is an unstable equilibrium.

(c) Consider the two level system. Assume conditions on $\nu_{0}$, namely on the density at 1 of the total mass of types 2 and 3 (as in Theorem 5) and that $v_{M}>2$. Then for sufficiently large $v_{2}$ type 1 becomes extinct and the mutualistic types dominate. Moreover they continue to dominate even if the level II selection ends, that is, $v_{2}(t)=1_{[0, T]}(t) v_{2}$ for sufficiently large $T$.

In this case we have a two stage process

Stage 1: Ignoring the mutualistic effect, survival and equilibria of altruistic types 2,3 by level II selection.

Stage 2: Takeover by the mutualistic pair 2,3.

Proof (a,b) Using the dual as above we can obtain the following equations by decomposing the first transitions of the dual into the different cases (recall (3.47)):

$$
\frac{d x_{1}(0)}{d t}=x_{1}(0)\left[\left(1-x_{1}(0)\right)-\frac{v_{m}}{2}\left(1-x_{1}(0)\right)^{2}\right] .
$$

The result follows by checking the sign of the derivative.

(c) By Theorem 5, in the two level system, under appropriate conditions on the density of the total mass of types 2 and 3 at 1 , for sufficiently large $v_{2}$ there is an equilibrium with mean mass of types 2,3 greater than $\frac{2}{v_{M}}$, that is $1-x_{1}(0)>\frac{2}{v_{M}}$, and therefore by part (b) type 1 becomes extinct and the mutualistic types dominate. Moreover they continue to dominate even if the level II selection ends.

Remark 7 Since the mutualistic pair can then persist even in the absence of further level II selection in (c), this illustrates the possible role of transient group selection in the long time genetic structure of a population. Without the role of level II selection, the simultaneous emergence of both types with at least critical density would be an event of higher order rarity.

Remark 8 We can also consider a random version of this. In this case we assume that $\nu_{0}=$ $\delta_{(1,0,0)}, \gamma_{1}>0, m_{12}=m_{13}=\varepsilon>0$. Then again for sufficiently large $v_{M}$ and $v_{2}$ type 1 dies out and the mutualistic types take over. The dual analysis involves four classes of selection birth events corresponding to the level I fitness of type 1, the mutualist fitnesses of types 2,3, the level II fitness of sites containing types 2 and 3 and level I coalescence. This will be carried out elsewhere.

\subsubsection{A three level system}

Consider a system with state space $\mathcal{P}(\mathcal{P}(\mathcal{P}(\mathbb{I}))$ ). For example, this could model a system of competing regions in which regions contain competing towns and each town contains a population with type space $\mathbb{I}$. The relative fitness $V_{3}(\nu)$, of a region (level III fitness) is assumed to depend on the distribution $\nu$ of the characteristics of the towns it contains so that $V_{3}:(\mathcal{P}(\mathcal{P}(\mathbb{I}))) \rightarrow[0,1]$

$$
V_{3}(\nu)=\prod_{k=1}^{K}\left[\int h_{k}\left(\mu_{k}\right) \nu\left(d \mu_{k}\right)\right]
$$


where $h_{k}$ is of the form (2.20), and convex combinations of functions of this form. We also allow individuals to migrate to a different region and even entire towns to move to a different region. Then the combined effect of three levels of selection can lead to complex behaviour which can be analyzed using the set-valued dual with values in $\left((\mathcal{I})^{\otimes \mathbb{N}}\right)^{\otimes \mathbb{N}}$.

\subsection{The study of more complex multilevel interactions - set-valued Monte Carlo approximation}

For more complex multilevel interactions it is natural to consider simulations since closed form solutions cannot be expected. However the numerical simulation of systems of Fleming-Viot processes involves the solutions of systems of nonlinear stochastic partial differential equations with degenerate boundary behaviour. The numerical simulation of such systems of stochastic differential equations is difficult. On the other hand the dual formulation involves only the simulation of continuous time Markov chains with discrete states which are easy to simulate by Monte Carlo methods. In particular one can compute means and covariances directly by simulating the dual process and determining the empirical distribution of the outcomes, namely, of the absorption probabilities (for equilibria) or equilibrium probabilities for fixation probabilities.

\subsection{Extensions}

The study of multilevel evolutionary systems arises in evolutionary biology, ecology, virology, sociology, economics, etc. These give rise to a wide range of mechanisms and interactions. Some of these can be modeled by extensions of the models and methods described above. For example, the models and the set-valued dual representations can be extended to systems with countably many types, recombination and horizontal gene transfer, higher level interactions, random environments, multiple species, and measure-valued processes with jumps leading to duals with multiple coalescence.

\section{References}

[1] E. Akin (1983). Hopf bifurcation in the two locus genetic model, Memoirs AMS 284.

[2] K. Aoki (1982). A condition for group selection to prevail over counteracting individual selection, Evolution 36, 832-842.

[3] K. Aoki (1983). A quantitative genetic model of reciprocal altruism: a condtion for kin or group selection to prevail, Proc. NAS 80, 4065-4068.

[4] A.J. Arnold ans K. Fristrup (1982). The theory of evolution by natural selection: a hierarchical expansion, Paleobiology 8, 113-129.

[5] S.R. Athreya and J.M. Swart (2005), Branching-coalescing systems, Prob. Theory Relat. Fields 131, No. 3, 376-414.

[6] T.C. Bergstrom (2002). Evolution of social behaviour: individual and group selection, J. Economic Perspectives 1, 67-88. 
[7] P. Bijma, W.M. Muir, J.A.M. Van Arendonk (2007), Multilevel+ selection 1: Quantitative genetics of inheritance and response to selection, Genetics 175, 277-288.

[8] P. Bijma, W.M. Muir, E.D. Ellen, J.B. Wolf, J.A.M. Van Arendonk (2007), Multilevel selection 2: Estimating the genetic parameters determining inheritance and response to selection,, Genetics 175, 289-299.

[9] R. Boyd and P.J. Richerson (2010). Transmission coupling mechanisms: cultural group selection, The Royal Soc. Phil. Trans. B, 365. 3787-3795.

[10] G. Boza and S. Számadó (2010). Beneficial laggards: multilevel selection, cooperative polymorphism and division of labour in Threshold Public Good Games, Evolutionary Biology 10:336.

[11] R.M. Brandon and R.M. Burian (1984). Genes, organisms and populations, MIT Press.

[12] J. Courtean and S. Lessard (2004). Sex ratio evolution through group selection using diffusion approximation, J. Math. Biol. 48, 340-356.

[13] J. Cremer, A. Melbinger and E. Frey (2012). Growth dynamics and the evolution of cooperation in microbial populations, Nature Scientific Reports 2:281: DOI 10.1038/srep00281

[14] J.F. Crow and K. Aoki (1984). Group selection for a polygenic trait: estimating the degree of population subdivision, PNAS 81, 6073-6077.

[15] D. A. Dawson (1993), Measure-valued Markov Processes. In: École d'Été de Probabilités de Saint Flour XXI, Lecture Notes in Mathematics 1541, 1-261, Springer-Verlag.

[16] D.A. Dawson (1997). Hierarchical and mean-field stepping stone models, in Progress in Population Genetics and Human Evolution, eds. P. Donnelly and S. Tavaré, Springer, IMA Volume in Mathematics and its Applications vol. 87.

[17] D.A. Dawson (2010). Introductory lectures on stochastic population systems, Technical Report Series of the Laboratory for Research in Statistics and Probability, Carleton University and University of Ottawa, No. 451.

[18] D.A. Dawson and K.J. Hochberg (1982). Wandering random measures in the Fleming-Viot model, Annals of Probability 10 (1982), 554-580.

[19] D.A. Dawson and K.J. Hochberg (1991). A multilevel branching model, Advances in Applied Probability 23(1991), 701-715.

[20] D.A. Dawson, K.J. Hochberg and V. Vinogradov (1996). High-density limits of hierarchically structured branching-diffusing populations, Stochastic Processes and their Applications, 62, 191-222.

[21] D.A. Dawson and J. Gärtner (1998). Analytic aspects of multilevel large deviations, in Asymptotic Methods in Probability and Statistics (ed. B. Szyszkowicz), Elsevier, Amsterdam, 401-440. 
[22] D.A. Dawson and A. Greven (1993) Hierarchically interacting Fleming-Viot processes with selection and mutation: multiple space-time scale analysis and quasi equilibria, Electronic J. of Prob., vol. 4, paper 4, pages 1-81.

[23] D.A. Dawson, L.G. Gorostiza and A. Wakolbinger (2004). Hierarchical equilibria of branching populations, Electronic Journal of Probability Volume 9, paper 12, 2004, 316-381.

[24] D.A. Dawson and A. Greven (2014). Spatial Fleming-Viot models with selection and mutation, Lecture Notes in Mathematics 2092, Springer.

[25] D.A. Dawson and Y. Wu (1996). Multilevel multitype models of an information system, I.M.A. Volume 84, Classical and Modern Branching Processes, eds. K.B. Athreya and P. Jagers, Springer-Verlag, pages 57-72.

[26] A. Etheridge (1993). Limiting behaviour of two-level measure-branching, Adv. Appl. Prob. $25,773-782$.

[27] S. N. Ethier and T. G. Kurtz (1986/93), Markov processes, characterization and convergence, Wiley, New York.

[28] S. N. Ethier and T. G. Kurtz (1987), The infinitely-many-alleles-model with selection as a measure-valued diffusion, Lecture Notes in Biomath., vol. 70, Springer-Verlag, 72-86.

[29] A. Gardner and A. Graffen (2009). Capturing the superorganism: a formal theory of group adaptation, J. Evol. Biol. doi:10.1111/j

[30] M.E. Gilpin (1975). Group Selection in Predator-Prey Communities, Princeton Univ. Press.

[31] C.J. Goodnight (2005). Multilevel selection: the evolution of cooperation in non-kin groups, Population Ecology 47, 3-12.

[32] O. Görnerup and J.P.Crutchfield (2006). Objects that make objects: the population dynamics of structural complexity, J.R. Soc. Interface 3, 345-349.

[33] L.G. Gorostiza, K.J. Hochberg and A. Wakolbinger (1995). Persistence of a critical super-2 process, J. Appl. Prob. 32, 535-540.

[34] A. Greven, V. Limic and A. Winter (2005). Representation theoremes for interacting Moran models, interacting Fisher-Wright diffusions and applications, EJP 10, 1286-1358.

[35] W.D. Hamilton (1964). The genetical evolution of social behavior, I,II, J. of Theor. Biol. 7, $1-52$.

[36] I.A. Hanski and M.E. Gilpin (1997). Metapopulation biology: ecology, genetics and evolution, Academic Press.

[37] J. Henrich (2003). Cultural group selection, coevolutionary processes and large-scale cooperation, J. Econ. Beh. \& Organization 53, 3-35 and 127-143. 
[38] P. Hogeweg and N. Takeuchi (2003). Multilevel selection in models of prebiotic evolution: compartments and spatial organization, Origin of Life and Evolution of the Biosphere 33, 375-403.

[39] L. Keller (1999) Levels of selection in evolution, Princeton University Press.

[40] M. Kimura (1983). Diffusion model of intergroup selection, with special reference to evolution of an altruistic gene, Proc. Nat. Acad. Sci. 80, 6317-6321.

[41] M. Kimura (1986). Diffusion model of population genetics incorporating group selection, with special reference to an altruistic trait, Lecture Notes in Math. 1203, 101-118, Springer.

[42] M. Kimura (1984). Evolution of an altruistic trait through group selection studied by the diffusion equation method, IMA J. Math. Applied in Medicine and Biology 1, 1-15.

[43] T. Kulich and J. Flegr (2011). Positive effects of multiple gene control on the spread of altruism by group selection, J. Theor. Biol. 234. E. G.

[44] E.G. Leigh, Jr. (2010). The group selection controversy. Journal of Evolutionary Biology 23: $6-19$.

[45] L. Lehmann, L. Keller, S. West, D. Roze (2007). Group selection and kin selection: two concepts but one process, PNAS 104, no. 16, 6736-6739.

[46] S. Lessard (2009). Diffusion approximations for one locus multi-allele kin selection, mutation and random drift in group-structured populations, a unifying approach to selection in population models, J. Math. Biol.

[47] S. Lion and M. van Baalen (2008). Self-structuring in spatial evolutionary ecology, Ecology Letters 11, 277-295.

[48] S. Lion, V.A.A. Jansen and T. Day (2011). Evolution in structured populations: beyond the kin versus group debate, Trends in Ecology and Evolution, 26, 193-201.

[49] G.G. Lorentz (1963). The degree of approximation by polynomials with positive coefficients, Math. Anal. 151, 239-251.

[50] S. Luo, M. Reed, J.C. Mattingly and K. Koelle (2012). The impact of host immune status on the within-host and population dynamics of antigenic immune excape, J. Royal Soc. Interface.

[51] S. Luo (2013). A unifying framework reveals key properties of multilevel selection, J. Theor. Biol. 341, 41-52.

[52] S. Luo and J. Mattingly (2015). Scaling limits of a model for selection at two scales, arXiv:1507.00397v1.

[53] J. Maynard Smith (1964). Groups secelction and kin selection, Nature 201, 1145-1147.

[54] J. Maynard Smith (1976). Group Selection, The Quarterly Review of Biology 51, 277-283. 
[55] J. Maynard Smith (1998). The units of selection, Novartis Found. Symp. 213: 203-11.

[56] D.W. McShea and M.A. Changizi (2003). Three puzzles in hierarchical evolution, Integr. Comp. Biol. 43, 74-81.

[57] O. Nerman (1981). On the convergence of supercritical general ( $C$-M-J) branching processes, Zeitschrift f. Wahrscheinlichkeitsth. verw. Gebiete, 57, 365-395.

[58] M.A. Nowak (2006) Evolutionary Dynamics, Harvard Univ. Press

[59] M.A. Nowak, C.E. Tarnita and E.O. Wilson (2010) The evolution of eusociality, Nature 466, 1057-1062.

[60] M.A. Nowak, R. Highfield (2011) Supercooperators, Simon and Schuster.

[61] Y. Ogura and N. Shimakura (1987) Stationary solutions and their stability for Kimura's diffusion model with intergroup selection, J. Math. Kyoto Univ. 27-2, 305-347.

[62] S. Okasha (2003). Recent work on the levels of selection problem, Human Nature Review $3,349-356$.

[63] S. Okasha (2005). Maynard Smith on the levels of selection question, Biol. and Philosophy, Springer.

[64] S. Okasha (2009). Evolution and the Levels of Selection, Oxford Univ. Press.

[65] M. Perc, J. Gómez-Gardeñes, A. Szolnoki, L.M. Floria and Y. Moreno (2013). Evolutionary dynamics of group interactions on structured populations: a review, J.R. Soc. Interface 10, 20120997.

[66] J. Paulsson (2002). Multileveled selection on plasmid replication, Genetics 161, 1373-1384.

[67] D.M. Rand (2001) The units of selection on mitochondrial DNA, Annu. Rev. Ecol. Syst. $32,415-448$.

[68] H. Kern Reeve and Bert Hölldober (2007). The emergence of a superorganism through intergroup competition, PNAS 104, 9736-9740.

[69] D. Roze and R.E. Michod (2001) Mutation, multilevel selection and the evolution of propagule size during the origin of multicellularity, Amer. Naturalist 158, 638-653.

[70] J. van Veelen M, Garcia, M.W. Sabelis and M. Egas (2012). Group selection and inclusive fitness are not equivalent; the Price equation vs. models and statistics. J Theor Biol 299.

[71] R.H. Schonmann, R. Vincente and N. Caticha (2012). Altruism can proliferate through group/kin selection despite high random gene flow, arXiv: 1208.0863v2.

[72] T. Shiga (1987). Existence and uniqueness of solutions for a class of non-linear diffusion equations, J. Math. Kyoto Univ. 27, 195-215. 
[73] T. Shiga (1982), Continuous time multi-allelic stepping stone models in population genetics, J. Math Kyoto Univ. 22-1, 1-40.

[74] T. Shiga and Uchiyama (1986), Stationary states and the stability of the stepping stone model involving mutation and selection, Prob. Th. Rel. Fields 73, 87-117.

[75] N. Shimakura (1985). Existence and uniqueness of solutions for a diffusion model of intergroup selection, J. Math. Kyoto Univ. 25, 775-788.

[76] E. Sober and D.S. Wilson (1998). Unto Others: The Evolution and Psychology of Unselfish Behavior, Harvard University Press.

[77] E. Szathmáry and L. Demeter (1987). Group selection of early replicators and the origin of life. Journal of Theoretical Biology 128, 463-486.

[78] Stanford Encyclopedia of Philosophy (2012). Units and levels of Selection.

[79] N. Takeuchi and P. Hogeweg (2009), Multilevel selection in models of prebiotic evolution II, a direct comparison of compartmentalization and spatial self-organization, PLOS Comp. Biol. Vol 5, Issue 10, e100542.

[80] P.D. Taylor, A.J. Irwin and T. Day (2000) Inclusive fitness in finite deme-structured and stepping-stone populations, Selection 1-3, 153-163.

[81] P.D. Taylor, T. Day and G. Wild (2007). From inclusive fitness to fixation probability in homogeneous strucutred populations, J. Thoer. Biol. 249, 101-110.

[82] A. Traulsen, A.M. Sengupta and M.. Nowak (2005). Evolutionary dynamics on two levels, J. Theor. Biol. 235, 393-401.

[83] A. Traulsen and M.A. Nowak (2006) Evolution of cooperation by multilevel selection, Proc. N.A.S. 103, 10952-10955.

[84] A. Traulsen, J.C. Claussen and C. Hauert (2005). Coevolutionary dynamics: from finite to infinite populations, Phys. Rev. Letters 95, 238701-1 - 238701-4.

[85] P. Turchin, T.E. Currie, E.A.L. Turner and S. Gavrilets (2013), War, space and the evolution of Old World societies, PNAS 110, 16384-16389.

[86] J. Vaillancourt (1988). On the existence of random McKean-Vlasov limits for triangular arrays of exchangeable diffusions, Stochastic Anal. Appl. 6, 431-446.

[87] M. van Veelen, S. Luo and B. Simon (2014). A simple model of group selection that cannot be analyzed by inclusive fitness, J. Theor. Biol.

[88] M. van Veelen, J. Garcia, M.W. Sabelis and M. Egas (2012). Group selection and inclusive fitness are not equivalent: the Pric equation vd. models and statistics, J. Theor. Biol. 299, 64-80. 
[89] A.E. Vinogradov (2004). Evolution of genome size: multilevel selection, mutation bias or dynamical chaos, Current Opinion in Genetics and Development 14, 620-626.

[90] M.J. Wade et al (2010) Multilevel and kin selection in a connected world, Nature vol. 463, E8-E9.

[91] G.G. Williams (1966). Adaptation and Natural Selection: A Critique of Some Current Evolutionary Thought, Princeton Univ. Press.

[92] G.G. Williams (1975) Sex and Evolution, Princeton Univ. Press.

[93] D.S. Wilson (1975). A theory of group selection, PNAS 72, 143-146.

[94] D.S. Wilson and E.O. Wilson (2007). Rethinking the theoretical foundation of sociobiology, The Quarterly Review of Biology 82, 327-348.

[95] E.O. Wilson (1973). Group selection and its significance for ecology, Bioscience 23, 631-638.

[96] Y. Wu (1994). Asymptotic Behavior of the Two Level Measure Branching Process, Ann. Probab 22, 854-874.

[97] V.C. Wynne-Edwards (1962). Animal dispersion in relation to social behaviour, Oliver and Boyd.

[98] V.C. Wynne-Edwards (1986). Evolution through group selection, Blackwell. 